\title{
A RELATIVE BASIS FOR MIXED TATE MOTIVES OVER THE PROJECTIVE LINE MINUS THREE POINTS
}

\author{
ISMAEL SOUDÈRES
}

\begin{abstract}
In a previous work, the author built two families of distinguished algebraic cycles in Bloch-Kriz cubical cycle complex over the projective line minus three points.

The goal of this paper is to show how these cycles induce well-defined elements in the $\mathrm{H}^{0}$ of the bar construction of the cycle complex and thus generate comodules over this $\mathrm{H}^{0}$, that is a mixed Tate motives over the projective line minus three points.

In addition, it is shown that out of the two families only one is needed at the bar construction level. As a consequence, the author obtains that one of the family gives a basis of the tannakian Lie coalgebra of mixed Tate motives over $\mathbb{P}^{1} \backslash\{0,1, \infty\}$ relatively to the tannakian Lie coalgebra of mixed Tate motives over $\operatorname{Spec}(\mathbb{Q})$. This in turns provides a new formula for Goncharov motivic coproduct, which really should be thought as a coaction. This new presentation is explicitly controlled by the structure coefficients of Ihara action by special derivation on the free Lie algebra on two generators.
\end{abstract}

\section{Contents}

1. Introduction

1.1. Multiple polylogarithms and mixed Tate motives

1.2. Distinguished algebraic cycles over $\mathbb{P}^{1} \backslash\{0,1, \infty\}$

1.3. Main results

2. Bar and cobar adjunctions

2.1. Notation and convention

2.2. Bar/cobar adjunction: associative case

2.3. Bar/cobar adjunction: commutative algebras/Lie Coalgebras

2.4. An explicit map

3. Families of bar elements

3.1. Lie algebra, special derivations and Lyndon words

3.2. The dual setting : a coaction and a Lie coalgebra

3.3. A differential system for cycles

4. A relative basis for mixed Tate motive over $\mathbb{P}^{1} \backslash\{0,1, \infty\}$

4.1. Relations between bar elements

4.2. A Basis for the geometric Lie coalgebra

References

Date: July 30, 2018.

None of this would have been possible without M. Levine patience, his explanations and deep inputs. The author is grateful to the MPIM in Bonn for providing ideal working conditions and support during my stay there as a guest where this work has been accomplished. 


\section{INTRODUCTION}

1.1. Multiple polylogarithms and mixed Tate motives. For a tuple $\left(k_{1}, \ldots, k_{m}\right)$ of integers, the multiple polylogarithm is defined by:

$$
L i_{k_{1}, \ldots, k_{m}}(z)=\sum_{n_{1}>\cdots>n_{m}} \frac{z^{n_{1}}}{n_{1}^{k_{1}} \cdots n_{m}^{k_{m}}} \quad(z \in \mathbb{C},|z|<1) .
$$

This is one of the one variable version of multiple polylogarithms in many variables defined by Goncharov in Gon05.

When $k_{1} \geqslant 2$, the series converges as $z$ goes to 1 and one recovers the multiple zeta values

$$
\zeta\left(k_{1}, \ldots k_{m}\right)=L i_{k_{1}, \ldots, k_{m}}(1)=\sum_{n_{1}>\cdots>n_{m}} \frac{1}{n_{1}^{k_{1}} \cdots n_{m}^{k_{m}}} .
$$

The case $m=1$ recovers the classical polylogarithm and the value of Riemann zeta function at $k_{1}: \zeta\left(k_{1}\right)$.

The values of multiple polylogarithms are important in geometric as they naturally appear as periods, in the Hodge or motivic sense, of moduli spaces of curves in genus 0 ( $[$ Bro09 $)$; as periods of the fundamental groups of $\mathbb{P}^{1}$ minus a finite set of points ([DG05]). In number theory, Zagier's conjecture Zag91 predicts that regulators of number fields are linear combinations of polylogarithms at special points.

Bloch and Kriz in BK94 constructed algebraic avatars of classical polylogarithms. However this was part of a larger work proposing in 1994 a tannakian category $\operatorname{MTM}(F)$ of mixed Tate motives over a number field $F$. Their construction begins with the cubical complex computing higher Chow groups which in the case of $\operatorname{Spec}(F)$ is commutative differential graded algebra $\mathcal{N}_{F}$. The $\mathrm{H}^{0}$ of the bar construction $B\left(\mathcal{N}_{F}\right)$ is a Hopf algebra and they defined $\operatorname{MTM}(F)$ as

$$
\operatorname{MTM}(F)=\text { category of comodule over } \mathrm{H}^{0}\left(\mathcal{N}_{F}\right) \text {. }
$$

Spitzweck in Spi (as presented in [Lev05]) proved that this construction agrees with Voevodsky definition of motives Voe00 and Levine's approach to mixed Tate motive [Lev93. More recently, M. Levine generalized this approach in [Lev11] to any quasi projective variety $X$ over the spectrum of a field $\mathbb{K}$ such that

- the motive of $X$ is mixed Tate in Cisinski and Déglise category $D M(\mathbb{K})$,

- the motive of $X$ satisfies Beilinson-Soulé vanishing property.

In order to do so, Levine used the complex $\mathcal{N}_{X}^{q f, \bullet}$ of quasi-finite cycles over $X$ (Definition 3.11) instead of the original Bloch-Kriz complex. This modification has better functoriality property and allows a simpler definition of the product structure. Working over $\operatorname{Spec}(\mathbb{Q})$, Levine's work shows that for $X=\mathbb{P}^{1} \backslash\{0,1, \infty\}$ :

Theorem 1.1 ([Lev11][Section 6.6 and Corollary 6.6.2]). Let $x$ be a $\mathbb{Q}$-point of $\mathbb{P}^{1} \backslash\{0,1, \infty\}$. Let $G_{\mathbb{P}^{1} \backslash\{0,1, \infty\}}$ and $G_{\mathbb{Q}}$ denote the Spectrum of $\mathrm{H}^{0}\left(B\left(\mathcal{N}_{\mathbb{P}^{1} \backslash\{0,1, \infty\}}^{q f, \bullet}\right)\right)$ and $\mathrm{H}^{0}\left(B\left(\mathcal{N}_{\mathbb{Q}}^{q f, \bullet}\right)\right)$ respectively. Then there is a tannakian category of mixed Tate motives over $\mathbb{P}^{1} \backslash\{0,1, \infty\}$ :

$$
\operatorname{MTM}\left(\mathbb{P}^{1} \backslash\{0,1, \infty\}\right)=\text { category of comodule over } \mathrm{H}^{0}\left(\mathcal{N}_{\mathbb{P}^{1} \backslash\{0,1, \infty\}}^{q f, \bullet}\right) .
$$

Moreover there is a split exact sequence:

$$
1 \longrightarrow \pi_{1}^{m o t}\left(\mathbb{P}^{1} \backslash\{0,1, \infty\}, x\right) \longrightarrow G_{\mathbb{P}^{1} \backslash\{0,1, \infty\}}^{\stackrel{p^{*}}{\longrightarrow}} G_{\mathbb{Q}} \longrightarrow 1
$$


where $p$ is the structural morphism $p: \mathbb{P}^{1} \backslash\{0,1, \infty\} \longrightarrow \operatorname{Spec}(\mathbb{Q})$. In the above exact sequence $\pi_{1}^{\text {mot }}\left(\mathbb{P}^{1} \backslash\{0,1, \infty\}, x\right)$ denotes Deligne-Goncharov motivic fundamental group [DG05].

The exact sequence (10) is the motivic avatar of the short exact sequence for etale fundamental groups. M. Levine however did not produce any specific motives. In particular, he did not produce any specific element in $\mathrm{H}^{0}\left(B\left(\mathcal{N}_{\mathbb{P}^{1} \backslash\{0,1, \infty\}}^{q f, \bullet}\right)\right)$; a natural motive being then the comodule cogenerated by such an element.

1.2. Distinguished algebraic cycles over $\mathbb{P}^{1} \backslash\{0,1, \infty\}$. In order to describe explicitly some elements in $\mathrm{H}^{0}\left(B\left(\mathcal{N}_{\mathbb{P}^{1} \backslash\{0,1, \infty\}}^{q f, \bullet}\right)\right)$, a first possible step is to produce a family of degree 1 elements in $\mathcal{N}_{\mathbb{P}^{1} \backslash\{0,1, \infty\}}^{q f, \bullet}$ which have a decomposable boundary inside the family. More explicitly, the differential of such an element is a linear combination of products of other elements inside the family.

In Sou12 the author produces such a family. Together with two explicit degree 1 weight 1 algebraic cycles generating the $\mathrm{H}^{1}\left(\mathcal{N}_{\mathbb{P}^{1} \backslash\{0,1, \infty\}}^{q f, \bullet}\right)$, the author obtains:

Theorem. For any Lyndon word $W$ in the letters $\{0,1\}$ of length $p \geqslant 2$, there exists a cycle $\mathcal{L}_{W}^{0}$ in $\mathcal{N}_{\mathbb{P}^{1} \backslash\{0,1, \infty\}}^{q f, 1}(p)$; i.e. a cycle of codimension $p$ in $\mathbb{P}^{1} \backslash\{0,1, \infty\} \times$ $\mathbb{A}^{2 p-1} \times A^{p}$ dominant and quasi-finite over $\mathbb{P}^{1} \backslash\{0,1, \infty\} \times \mathbb{A}^{2 p-1}$.

$\mathcal{L}_{W}^{0}$ satisfies :

- $\mathcal{L}_{W}^{0}$ has a decomposable boundary,

- $\mathcal{L}_{W}^{0}$ admits an equidimensional extension to $\mathbb{A}^{1}$ with empty fiber at 0 .

A similar statement holds for 1 in place of 0 .

The above result relies on

- The dual of the action of the free Lie algebra on two generators on itself by Ihara special derivations in order to "guess" the differential of cycles $\mathcal{L}_{W}^{0}$.

- The pull-back by the multiplication $\mathbb{A}^{1} \times A^{1} \longrightarrow \mathbb{A}^{1}$ in order to build the cycles $\mathcal{L}_{W}^{0}$ from their boundaries.

The free Lie algebra on two generators $\operatorname{Lie}\left(X_{0}, X_{1}\right)$ is the Lie algebra associated to $\pi_{1}^{\text {mot }}\left(\mathbb{P}^{1} \backslash\{0,1, \infty\}, x\right)$ from the exact sequence (1) and hence appears naturally in the construction. However its graded dual $Q_{\text {geom }}$, which is more closely related to $\mathrm{H}^{0}\left(B\left(\mathcal{N}_{\mathbb{P}^{1} \backslash\{0,1, \infty\}}^{q f, \bullet}\right)\right)$, is more natural in our context. It appears in the sequence dual to (1) :

$$
0 \longrightarrow Q_{\mathbb{Q}} \longrightarrow Q_{\mathbb{P}^{1} \backslash\{0,1, \infty\}} \longrightarrow Q_{\text {geom }} \longrightarrow 0
$$

where $Q_{\mathbb{P}^{1} \backslash\{0,1, \infty\}}$ and $Q_{\mathbb{Q}}$ denote respectively the set of indecomposable elements of $\mathrm{H}^{0}\left(B\left(\mathcal{N}_{\mathbb{P}^{1} \backslash\{0,1, \infty\}}^{q f, \bullet}\right)\right)$ and $\mathrm{H}^{0}\left(B\left(\mathcal{N}_{\operatorname{Spec}(\mathbb{Q})}^{q f, \bullet}\right)\right)$.

1.3. Main results. In this paper, using the unit of the adjunction between bar and cobar construction in the commutative/coLie case, we lift the above algebraic cycle to elements in $Q_{\mathbb{P}^{1} \backslash\{0,1, \infty\}}$ viewed as a subspace of $\mathrm{H}^{0}\left(B\left(\mathcal{N}_{\mathbb{P}^{1} \backslash\{0,1, \infty\}}^{q f, \bullet}\right)\right)$ by the mean of Hain's projector $p_{\text {II }}$ (see [Hai86] or Section 2.3.2). Let $\pi_{1}: B\left(\mathcal{N}_{\mathbb{P}^{1} \backslash\{0,1, \infty\}}^{q f, \bullet}\right) \longrightarrow$ $\mathcal{N}_{X}^{q f,} \bullet$ the projection onto the tensor degree 1 part of the bar construction. We obtain

Theorem (Theorem 3.16). For any Lyndon word $W$ of length $p \geqslant 2$ there exist elements $\mathcal{L}_{W}^{B}$, in the bar construction $B\left(\mathcal{N}_{\mathbb{P}^{1} \backslash\{0,1, \infty\}}^{q f, \bullet}\right.$ satisfying:

- One has $\pi_{1}\left(\mathcal{L}_{W}^{B}\right)=\mathcal{L}_{W}^{0}$.

- It is in the image of $p_{\mathrm{II}}$; that is in $Q_{\mathbb{P}^{1} \backslash\{0,1, \infty\}}$;

- It is of degree 0 and map to 0 under the bar differential; it induces a class in $\mathrm{H}^{0}\left(B_{\mathbb{P}^{1} \backslash\{0,1, \infty\}}\right)$ and in $\mathrm{H}^{0}\left(Q_{\mathbb{P}^{1} \backslash\{0,1, \infty\}}\right)$. 
- Its cobracket in $Q_{\mathbb{P}^{1} \backslash\{0,1, \infty\}}$ is given by the differential of $\mathcal{L}_{W}^{0}$.

A similar statement holds for cycles $\mathcal{L}_{W}^{1}$ and constant cycles $\mathcal{L}_{W}^{0}(1)$ induced by the fiber at 1 of $\mathcal{L}_{W}^{0}$ (after extension to $\mathbb{A}^{1}$ ).

Then we show that the elements $\mathcal{L}_{W}^{B}$ and $\mathcal{L}_{W}^{1, B}$ are related:

Theorem (Theorem 4.4). For any Lyndon word $W$ of length $p \geqslant 2$ the following relation holds in $\mathrm{H}^{0}\left(Q_{X}\right)=Q_{\mathrm{H}^{0}\left(B_{X}\right)}$

$$
\mathcal{L}_{W}^{B}-\mathcal{L}_{W}^{1, B}=\mathcal{L}_{W}^{B}(1) .
$$

The proof relies on the relation between the situation on $\mathbb{P}^{1} \backslash\{0,1, \infty\}$ and on $\mathbb{A}^{1}$ where the cohomology of $\mathcal{N}_{\mathbb{A}^{1}}^{q f,} \bullet$ is given by constant cycle because of $\mathbb{A}^{1}$ homotopy invariance of higher Chow groups. As a corollary one obtains a description of the cobracket of $\mathcal{L}_{W}^{B}$ in terms of the structure coefficients of Ihara action by special derivation. This makes explicit the relation between the dual of Ihara action (or bracket) and Goncharov motivic coproduct which here, as in Brown Bro12, is in fact a coaction. In a group setting, Goncharov coproduct is really the action of $G_{\mathbb{Q}}$ on $\pi_{1}^{m o t}\left(\mathbb{P}^{1} \backslash\{0,1, \infty\}, x\right)$ induced by the short exact sequence (1).

We conclude the paper by showing at Theorem 4.8 that the family of elements $\mathcal{L}_{W}^{B}$ induces a basis of $Q_{\text {geom }}$; that is a basis of $Q_{\mathbb{P}^{1} \backslash\{0,1, \infty\}}$ relatively to $Q_{\mathbb{Q}}$.

Our methods are structural and geometric by opposition to Gangl Goncharov and Levin approach [GGL09] toward lifting cycles to bar elements using the combinatorics of "rooted polygons".

The paper is organized as follow

- In the next section, Section 2, we begin by a short review of differential graded (dg) vector spaces and formalism. Then we present the bar cobar adjunction in the case of associative algebras and coalgebras and in the case of commutative algebras and Lie coalgebras.

- In Section 3, we present the action of $\operatorname{Lie}\left(X_{0}, X_{1}\right)$ on itself by Ihara's special derivations and the corresponding Lie coalgebra. From there we recall the result from [Sou12] constructing the cycles $\mathcal{L}_{W}^{\varepsilon}$. We conclude this section by lifting the cycle to elements in the bar construction.

- In section 4.1, we prove that bar elements $\mathcal{L}_{W}^{B}$ and $\mathcal{L}_{W}^{1, B}$ are equal up to the constant (over $\mathbb{P}^{1} \backslash\{0,1, \infty\}$ ) bar element $\mathcal{L}_{W}^{B}(1)$. From there we make explicit the relation with Ihara's coaction and prove that the elements $\mathcal{L}_{W}^{B}$ provides a basis for $\mathbb{Q}_{\text {geom }}$ graded dual of the Lie algebra associated to $\pi_{1}^{m o t}\left(\mathbb{P}^{1} \backslash\{0,1, \infty\}, x\right)$.

\section{BAR AND COBAR ADJUNCTIONS}

In this section we recall how the bar/cobar constructions gives a pair of adjoint functors in the two following cases:

and

$$
B:\left\{\begin{array}{c}
\text { diff. gr. ass. } \\
\text { algebras }
\end{array}\right\} \rightleftharpoons\left\{\begin{array}{c}
\text { diff. gr. coass. } \\
\text { coalgebras }
\end{array}\right\}: \Omega
$$

$$
B_{c o m}:\left\{\begin{array}{c}
\text { diff. gr. com. } \\
\text { ass. algebras }
\end{array}\right\} \rightleftharpoons\left\{\begin{array}{c}
\text { diff. gr. coLie. } \\
\text { coalgebras }
\end{array}\right\}: \Omega_{c o L} .
$$

As a differential graded commutative algebras $A$ is also an associative algebra, we will recall how the two constructions are related in this case.

The material developed here is well known and can be found in GinzburgKapranov GK94 work even if their use of graded duals replaces coalgebra structures by algebra structures. The presentation used here is closer to the Kozul duality as developed by Jones and Getzler in GJ94. We follow here the signs conventions 
and the formalism presented by Loday and Vallette in [LV12. The associative case is directly taken from [LV12, Chap. 2] in a cohomological version. More about the commutative/coLie adjunction can be found in [GJ94, GK94, Mil12, SW11a].

\subsection{Notation and convention.}

2.1.1. Koszul sign rule. The objects are all object of the category of (sign) graded $\mathbb{Q}$-vector spaces. The degree of an homogeneous element $v$ in $V$ is denoted by $|v|$ or $|v|_{V}$ if we want to emphasis where $v$ is. The symmetric structure is given by the switching map

$$
\tau: V \otimes W \longrightarrow W \otimes V, \quad \tau(v \otimes w)=(-1)^{|v||w|} w \otimes v .
$$

For any maps $f: V \rightarrow V^{\prime}$ and $g: W \rightarrow W^{\prime}$ of graded spaces, the tensor product

$$
f \otimes g: V \otimes W \longrightarrow V^{\prime} \otimes W^{\prime}
$$

is defined by

$$
(f \otimes g)(v \otimes w)=(-1)^{|g||v|} f(v) \otimes g(w) .
$$

A differential graded $(\mathrm{dg})$ vector space is a graded vector space equipped with a differential $d_{V}$ (or simply $d$ ); that is a degree 1 linear map satisfying $d_{V}^{2}=0$. For $V$ and $W$ two dg vector spaces the differential on $V \otimes W$ is defined by

$$
d_{V \otimes W}=\mathrm{id}_{V} \otimes d_{W}+d_{V} \otimes \mathrm{id}_{W} .
$$

Definition 2.1. Let $V=\oplus_{n} V^{n}$ and $W=\oplus_{n} W^{n}$ be two graded vector spaces. A morphism of degree $r$, say $f: V \longrightarrow W$, is a collection of morphisms $f_{n}: V^{n} \longrightarrow$ $W^{n+r}$. Let $\operatorname{Hom}(V, W)_{r}$ be the vector space of morphisms of degree $r$.

When $V$ and $W$ are dg vector spaces, the graded vector space $\operatorname{Hom}(V, W)=$ $\oplus \operatorname{Hom}(V, W)_{r}$ turns into a a dg vector space with differential given by :

$$
d_{\text {Hom }}(f)=d_{W} \circ f-(-1)^{r} f \circ d_{V}
$$

for any homogeneous element $f$ of degree $r$. A $d g$ morphism $f: V \longrightarrow W$ is a morphism satisfying $d_{\text {Hom }}(f)=0$.

The dual of a graded vector space $V=\oplus_{n} V^{n}$ is defined by

$$
V^{*}=\oplus_{n} \operatorname{Hom}_{V e c t}\left(V^{-n}, \mathbb{Q}\right)=\operatorname{Hom}(V, N)
$$

where the dg vector space $N$ is defined by $N=\mathbb{Q}$ concentrated in degree 0 with 0 differential. One has an obvious notion of cohomology on dg vector space.

Definition 2.2 ((de)suspension). Let $S=s \mathbb{Q}$ be the 1 dimensional dg vector space concentrated in degree 1 (that is $d_{S}=0$ ) generated by $s$.

The dual of $S$ is a one dimensional dg vector space denoted by $S^{-1}$ and generated by a degree -1 element $s^{-1}$ dual to $s$.

Let $V, d_{V}$ be a dg vector space. Its suspension $\left(s V, d_{s V}\right)$, is the $\mathrm{dg}$ vector space $S \otimes V$. Its desuspension $\left(s^{-1} V, d_{s^{-1} V}\right)$ is $S^{-1} \otimes V$.

There is a canonical identification $V^{n-1} \simeq(s V)^{n}$ given by

$$
i_{s}: V \longrightarrow s V \quad v \longmapsto(-1)^{|v|_{V}} s \otimes v ;
$$

under this identification $d_{s V}=-d_{V}$. 
2.1.2. Associative dg algebra. A differential graded associative algebra $\left(A, d_{A}\right)$ abbreviated into dga algebra is a dg vector space equipped with a unital associative product $\mu_{A}$ of degree 0 commuting with the differential :

$$
d_{A} \circ \mu_{A}=\mu_{A} \circ d_{A \otimes A}
$$

and satisfying the usual commutative diagrams for an associative algebra, all the maps involved being maps of dg vector spaces.

This last equality is nothing but Leibniz rule. The unit $1_{A}$ belongs to $A^{0}$. On elements, one writes $a \cdot{ }_{A} b$ or simply $a \cdot b$ instead of $\mu_{A}(a \otimes b)$.

Definition 2.3. The $\operatorname{dga} A$ is connected if $A^{0}=1_{A} \mathbb{Q}$.

Definition 2.4 (Tensor algebra). The tensor algebra over a dg vector space $V$ is defined by

$$
T(V)=\bigoplus_{n \geqslant 0} V^{\otimes n}
$$

and equipped with the differential induced on each $V^{\otimes n}$ by $d_{V}$ and with the concatenation product given by

$$
\left[a_{1}|\cdots| a_{n}\right] \otimes\left[a_{n+1} \mid \cdots a_{n+m}\right] \longmapsto\left[a_{1}|\cdots| a_{n}\left|a_{n+1}\right| \cdots a_{n+m}\right]
$$

where the "bar" notation $\left[a_{1}|\cdots| a_{n}\right]$ stands for $a_{1} \otimes \cdots \otimes a_{n}$ in $V^{\otimes n}$.

Note that the degree of $\left[a_{1}|\cdots| a_{n}\right]$ is $\left|a_{1}\right|_{V}+\cdots\left|a_{n}\right|_{V}$ and that $T(V)$ admits a natural augmentation given by $\varepsilon\left(\left[a_{1}|\cdots| a_{n}\right]\right)=0$ for $n>0$ and the convention $V^{\otimes 0}=\mathbb{Q}$. The concatenation product is associative.

2.1.3. Commutative and anti commutative algebras. A commutative dga algebra $\left(A, d_{A}, \mu_{A}\right)$ or cdga algebra is a dga algebra such that the multiplication commutes with the switching map:

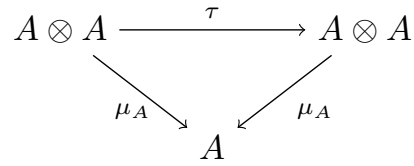

On homogeneous elements, this reads as

$$
a \cdot b=(-1)^{|a||b|} b \cdot a .
$$

Let $V$ be a dg vector space and $n \geqslant 1$ an integer. The symmetric group $\mathbb{S}_{n}$ acts on $V^{\otimes n}$ in two natural ways : the symmetric action $\rho_{S}$ and the antisymmetric action $\rho_{\Lambda}$ (both graded).

For $i$ in $\{1, \ldots n-1\}$, let $\tau_{i}$ be the permutation exchanging $i$ and $i+1$. It is enough to define both actions for the $\tau_{i}$ :

$$
\rho_{S}\left(\tau_{i}\right)=\underbrace{\mathrm{id} \otimes \cdots \mathrm{id}}_{i-1 \text { factors }} \otimes \tau \otimes \mathrm{id} \otimes \cdots \otimes \mathrm{id}
$$

and

$$
\rho_{\Lambda}\left(\tau_{i}\right)=\underbrace{\mathrm{id} \otimes \cdots \mathrm{id}}_{i-1 \text { factors }} \otimes(-\tau) \otimes \mathrm{id} \otimes \cdots \otimes \mathrm{id} .
$$

where $\tau$ is the usual switching map. Both action involved signs. The graded signature $\varepsilon^{g r}(\sigma) \in\{ \pm 1\}$ of a permutation $\sigma$ is defined by

$$
\rho_{S}(\sigma)\left(v_{1} \otimes \cdots \otimes v_{n}\right)=\varepsilon^{g r}(\sigma) v_{\sigma^{-1}(1)} \otimes \cdots \otimes v_{\sigma^{-1}(n)} .
$$

Then, one has

$$
\rho_{\Lambda}(\sigma)\left(v_{1} \otimes \cdots \otimes v_{n}\right)=\varepsilon(\sigma) \varepsilon^{g r}(\sigma) v_{\sigma^{-1}(1)} \otimes \cdots \otimes v_{\sigma^{-1}(n)}
$$


where $\varepsilon(\sigma)$ is the usual signature. Let $p_{S, n}$ be the projector defined on $V^{\otimes n}$ by

$$
p_{S, n}=\frac{1}{n !}\left(\sum_{\sigma \in \mathbb{S}_{n}} \rho_{S}(\sigma)\right) \text {. }
$$

Definition 2.5. The (graded) symmetric algebra $S^{g r}(V)$ over $V$ is defined as the quotient of $T(V)$ by the two side ideal generated by (id $-\tau)(a \otimes b)$.

One can write

$$
S^{g r}(V)=\bigoplus_{n \geqslant 0} S^{g r, n}(V)
$$

where $S^{g r, n}(V)=V^{\odot n}$ is the quotient of $V^{\otimes n}$ by the symmetric action of $\mathbb{S}_{n}$.

One also has the isomorphism $S^{g r, n}(V)=p_{S, n}\left(V^{\otimes n}\right) . S^{g r}(V)$ is the free commutative algebra over $V$. We may write simply $V \odot V$ for $S^{g r, 2}(V)$.

Definition 2.6. The (graded) antisymmetric algebra $\Lambda^{g r}(V) V$ is defined as the quotient of $T(V)$ by the two side ideal generated by (id $+\tau)(a \otimes b)$.

One can write

$$
\Lambda^{g r}(V)=\bigoplus_{n \geqslant 0} \Lambda^{g r, n}(V)
$$

where $\Lambda^{g r, n}(V)=V^{\wedge n}$ is the quotient of $V^{\otimes n}$ by the antisymmetric action of $\mathbb{S}_{n}$. We may write $V \wedge V$ for $\Lambda^{g r, 2}(V)$.

As in the symmetric case, $\Lambda^{g r}(V)$ is also the image of $V^{\otimes n}$ by the projector $p_{\Lambda, n}=1 /(n !)\left(\sum_{\sigma} \rho_{\Lambda}(\sigma)\right)$ and $\Lambda(V)$ is the free antisymmetric algebra over $V$.

2.1.4. Associative coalgebra. A differential graded associative coalgebra $\left(C, d_{C}\right)$ abbreviated into dga coalgebra is a dg vector space equipped with a counital associative coproduct $\Delta_{C}$ of degree 0 commuting with the differential :

$$
d_{C \otimes C} \circ \Delta_{C}=\Delta_{C} \circ d_{C}
$$

and satisfying the usual commutative diagrams for an associative coalgebra, all the maps involved being maps of dg vector spaces.

The iterated coproduct $\Delta^{n}: C \longrightarrow C^{\otimes(n+1)}$ is

$$
\Delta^{n}=(\Delta \otimes \text { id } \otimes \cdots \otimes \text { id }) \Delta^{n-1} \quad \text { and } \quad \Delta^{1}=\Delta .
$$

This definition is independent of the place of the $\Delta$ factor (here in first position) because of the associativity of the coproduct. We Will use Sweedler's notation:

$$
\Delta(x)=\sum x_{(1)} \otimes x_{(2)}, \quad(\Delta \otimes \mathrm{id}) \Delta(x)=\sum x_{(1)} \otimes x_{(2)} \otimes x_{(3)}
$$

and

$$
\Delta^{n}(x)=\sum x_{(1)} \otimes \cdots \otimes x_{(n+1)} .
$$

A coaugmentation on $C$ is a morphism of dga coalgebra $u: \mathbb{Q} \rightarrow C$. In this case, $C$ is canonically isomorphic to $\operatorname{ker}(\varepsilon) \oplus \mathbb{Q} u(1)$. Let $\bar{C}=\operatorname{ker}(\varepsilon)$ be the kernel of the counit.

When $C$ is coaugmented, the reduced coproduct is $\bar{\Delta}=\Delta-1 \otimes \mathrm{id}-\mathrm{id} \otimes 1$. It is associative and there is an iterated reduced coproduct $\bar{\Delta}^{n}$ for which we also use Sweedler's notation.

Definition 2.7. $C$ is conilpotent when it is coaugmented and when, for any $x$ in $C$, one has $\bar{\Delta}^{n}(x)$ vanishes for $n$ large enough.

A cofree associative dga coalgebra over the dg vector space is by definition a conilpotent dga coalgebra $F^{c}(V)$ equipped with a linear map of degree $0 p$ : $F^{c}(V) \longrightarrow V$ commuting with the differential such that $p(1)=0$. It factors any morphism of dg vector space $\phi: C \longrightarrow V$ where $C$ is a conilpotent dga coalgebra with $\phi(1)=0$. 
Definition 2.8 (Tensor coalgebra). The tensor coalgebra over $V$ is defined by

$$
T^{c}(V)=\bigoplus_{n \geqslant 0} V^{\otimes n}
$$

and equipped with the differential induced on each $V^{\otimes n}$ by $d_{V}$ and with the deconcatenation coproduct given by

$$
\left[a_{1}|\cdots| a_{n}\right] \longmapsto \sum_{i=0}^{n+1}\left[a_{1}|\cdots| a_{i}\right] \otimes\left[a_{i+1} \mid \cdots a_{n}\right] .
$$

The deconcatenation coproduct is associative. The natural projection $\pi_{V}$ : $T^{c}(V) \longrightarrow \mathbb{Q}=V^{\otimes 0}$ onto the tensor degree 0 part is a counit for $T^{c}(V)$ while the inclusion $\mathbb{Q}=V^{\otimes 0} \longrightarrow T^{c}(V)$ gives the coaugmentation. The tensor coalgebra $T^{c}(V)$ is the cofree counital dga coalgebra over $V$.

2.1.5. $d g$ Lie algebra. We review here the definition of Lie algebra and Lie coalgebra in the $\mathrm{dg}$ formalism. For any $\mathrm{dg}$ vector space $V$, let $\xi$ be the cyclic permutation of $V \otimes V \otimes V$ defined by

$$
\xi=(\mathrm{id} \otimes \tau)(\tau \otimes \mathrm{id}) .
$$

It corresponds to the cycle sending 1 to 3,3 to 2 and 2 to 1 .

Definition 2.9. A dg Lie algebra $L$ is a $\mathrm{dg}$ vector space equipped with a degree 0 map of dg vector spaces $c: L \otimes L \longrightarrow L$ ( $c$ stands for "crochet") satisfying

$$
c \circ \tau=-c \quad \text { and } \quad c \circ(c \otimes \text { id }) \circ\left(\text { id }+\xi+\xi^{2}\right)=0 .
$$

On elements, we will use a bracket notation $[x, y]$ instead of $c(x \otimes y)$.

In the above definition, the first relation is the usual antisymmetry of the bracket which gives in the dg context:

$$
[x, y]=(-1)^{|x||y|}[y, x] .
$$

The second relation is the Jacobi relation:

$$
[[x, y], z]+(-1)^{|x|(|y|+|z|)}[[y, z], x]+(-1)^{|z|(|y|+|x|)}[[z, x], y]=0 .
$$

One remarks that $(c \otimes$ id $) \circ \xi=\tau \circ($ id $\otimes c)$ and that $(c \otimes$ id $) \circ \xi^{2}=((c \circ \tau) \otimes$ id $) \circ($ id $\otimes \tau)$. Using this and the antisymmetry relation, one can rewrite the Jacobi relation as a Leibniz relation:

$$
c \circ(c \otimes \text { id })=c \circ(\text { id } \otimes c)+c \circ(c \otimes \text { id }) \circ(\text { id } \otimes \tau) .
$$

The definition of a dg Lie coalgebra is dual to the definition of a Lie algebra.

Definition 2.10. A dg Lie coalgebra $\mathcal{L}^{c}$ is a dg vector space equipped with a degree 0 map of dg vector spaces $\delta: \mathcal{L}^{c} \longrightarrow \mathcal{L}^{c} \otimes \mathcal{L}^{c}$ satisfying

$$
\tau \circ \delta=-\delta \quad \text { and } \quad\left(\text { id }+\xi+\xi^{2}\right) \circ(\delta \otimes \mathrm{id}) \circ \delta=0
$$

The first condition shows that $\delta$ induces a map (again denoted by $\delta$ )

$$
\delta: \mathcal{L}^{c} \longrightarrow \mathcal{L}^{c} \wedge \mathcal{L}^{c} \text {. }
$$

Let $\tau_{12}: \mathcal{L}^{c \otimes 3} \longrightarrow \mathcal{L}^{c \otimes 3}$ be the permutation exchanging the two first factors. The second condition show that the following diagram is commutative

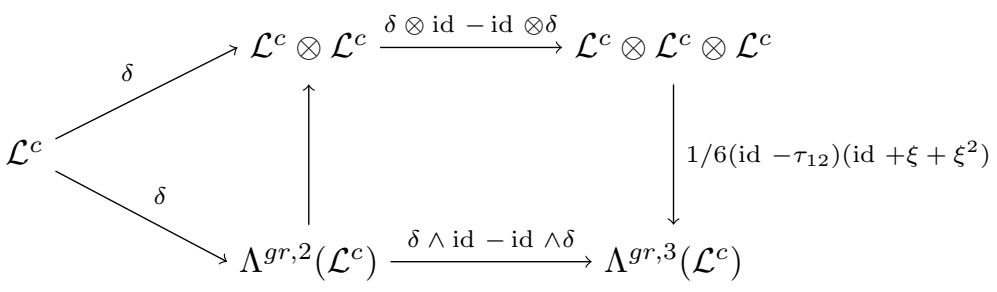


and that the composition going through the bottom line is 0 .

\subsection{Bar/cobar adjunction: associative case.}

2.2.1. Bar construction. In this subsection, we recall briefly the bar/cobar construction and how they give a pair of adjoint functor in the associative case.

$$
B:\left\{\begin{array}{c}
\text { diff. gr. ass. } \\
\text { algebras }
\end{array}\right\} \rightleftharpoons\left\{\begin{array}{c}
\text { diff. gr. coass. } \\
\text { coalgebras }
\end{array}\right\}: \Omega .
$$

Let $\left(A, d_{A}, \mu_{A}, \varepsilon_{A}\right)$ be an augmented dga algebra and $\bar{A}=\operatorname{ker}\left(\varepsilon_{A}\right)$ its augmentation ideal. The bar construction of $A$ is obtained by twisting the differential of the dga free coalgebra $T^{c}\left(s^{-1} \bar{A}\right)$.

The differential $d_{A}$ makes $\bar{A}$ and thus $s^{-1} \bar{A}$ into a $\mathrm{dg}$ vector vector space. Let $D_{1}$ denote the induced differential on $T^{c}\left(s^{-1} \bar{A}\right)$ which in tensor degree $n$ is:

$$
\sum_{i=1}^{n} \mathrm{id}^{i-1} \otimes d_{s^{-1} \bar{A}} \otimes \mathrm{id}^{n-i} \text {. }
$$

$S^{-1}=s^{-1} \mathbb{Q}$ admits an associative product-like map of degree +1 defined by:

$$
\Pi_{s}: s^{-1} \mathbb{Q} \otimes s^{-1} \mathbb{Q} \longrightarrow s^{-1} \mathbb{Q} \quad \Pi_{s}\left(s^{-1} \otimes s^{-1}\right)=s^{-1} .
$$

The map $\Pi_{s}$ and the restriction $\mu_{\bar{A}}$ of the multiplication $\mu_{A}$ to $\bar{A}$ induce the following map:

$$
f: s^{-1} \mathbb{Q} \otimes \bar{A} \otimes s^{-1} \mathbb{Q} \otimes \bar{A} \stackrel{\mathrm{id} \otimes \tau \otimes \mathrm{id}}{\longrightarrow} s^{-1} \mathbb{Q} \otimes s^{-1} \mathbb{Q} \otimes \bar{A} \otimes \bar{A} \stackrel{\Pi_{s} \otimes \mu_{\bar{A}}}{\longrightarrow} s^{-1} \mathbb{Q} \otimes \bar{A} .
$$

This map induces a degree 1 map $D_{2}: T^{c}\left(s^{-1} \bar{A}\right) \longrightarrow T^{c}\left(s^{-1} \bar{A}\right)$ which satisfies $D_{2}^{2}=0$ because of the associativity of $\mu_{A}$

One check that the degree 1 morphisms $D_{1}$ and $D_{2}$ commute (in the graded sense):

$$
D_{1} \circ D_{2}+D_{2} \circ D_{1}=0
$$

The coproduct on $T^{c}\left(s^{-1} \bar{A}\right)$ is given by the deconcatenation coproduct. From these definitions, one obtains (see [LV12][Section 2.2.1]) the following.

Lemma 2.11. The complex $B(A)=\left(T^{c}\left(s^{-1} \bar{A}\right), d_{B}\right)$ with $d_{B}=D_{1}+D_{2}$ and endowed with the deconcatenation coproduct $\Delta$ is a conilpotent dga coalgebra.

We recall below the explicit formulas related to the bar construction $B(A)$ :

- An homogeneous element a of tensor degree $n$ is denoted by

$$
\left[s^{-1} a_{1}|\cdots| s^{-1} a_{n}\right]
$$

or when the context is clear enough not to forget the shifting simply by $\left[a_{1}|\cdots| a_{n}\right]$. Its degree is given by:

$$
\operatorname{deg}_{B}(\mathbf{a})=\sum_{i=1}^{n} \operatorname{deg}_{s^{-1} \bar{A}}\left(s^{-1} a_{i}\right)=\sum_{i=1}^{n}\left(\operatorname{deg}_{A}\left(a_{i}\right)-1\right)
$$

- the coproduct is given by:

$$
\Delta(\mathbf{a})=\sum_{i=1}^{n}\left[s^{-1} a_{1}|\cdots| s^{-1} a_{i}\right] \otimes\left[s^{-1} a_{i+1}|\cdots| s^{-1} a_{n}\right] .
$$

- Let $\eta_{\mathbf{a}}(i)$ or simply $\eta(i)$ denote the "partial degree" of a:

$$
\eta_{\mathbf{a}}(i)=\sum_{k=1}^{i} \operatorname{deg}_{s^{-1} \bar{A}}\left(s^{-1} a_{k}\right)=\sum_{k=1}^{i}\left(\operatorname{deg}_{A}\left(a_{k}\right)-1\right) .
$$


- The differential $D_{1}$ and $D_{2}$ are explicitly given by the formulas:

$$
D_{1}(\mathbf{a})=-\sum_{i=1}^{n}(-1)^{\eta(i-1)}\left[s^{-1} a_{1}|\cdots| s^{-1} d_{A}\left(a_{i}\right)|\cdots| s^{-1} a_{n}\right]
$$

and

$$
D_{2}(\mathbf{a})=-\sum_{i=1}^{n}(-1)^{\eta(i)}\left[s^{-1} a_{1}|\cdots| s^{-1} \mu_{A}\left(a_{i}, a_{i+1}\right)|\cdots| s^{-1} a_{n}\right] .
$$

The global minus sign in $D_{1}$ appears because the differential of the dg vector space $s^{-1} \bar{A}$ is given by $d_{s^{-1}} \bar{A}\left(s^{-1} a\right)=-s^{-1} d_{A}(a)$. The other signs are due to the Kozul sign rules taking care of the shifting.

Remark 2.12. This construction can be seen as a simplicial total complex associated to the complex $A$ (as in BK94). Here, the augmentation makes it possible to use directly $\bar{A}$ without referring to the tensor coalgebra over $A$ and without the need of killing the degeneracies. However the simplicial presentation usually masks the need of working with the shifted complex; in particular for sign issues.

The bar construction $B(A)$ also admits a product $m$ which shuffles the tensor factors. However, this extra structure becomes more interesting when $A$ is graded commutative and we will present it in the next section.

The bar construction is a quasi-isomorphism invariant as shown in [LV12] (Proposition 2.2.4) and the construction provides a functor:

$$
B:\{\text { aug. dga algebra }\} \longrightarrow\{\text { coaug. dga coalgebra }\} \text {. }
$$

2.2.2. Cobar construction. Analogously, one constructs the cobar functor. Let $\left(C, d_{C}, \Delta_{C}, \varepsilon_{C}\right)$ be a coaugmented dga coalgebra decomposed as $C=\bar{C} \oplus \mathbb{Q}$. Consider $T(s \bar{C})$ the free algebra over $s \bar{C}$ (with concatenation product). The differential on $C$ induces a differential $d_{1}$ on $T(s \bar{C}) . S=s \mathbb{Q}$ comes with a coproduct-like degree 1 map dual to $\Pi_{s}$ :

$$
\Delta_{s}: s \mathbb{Q} \longrightarrow s \mathbb{Q} \otimes s \mathbb{Q}, \quad \Delta_{s}(s)=-s \otimes s .
$$

The map $\Delta_{s}$ and the restriction of the reduced coproduct $\bar{\Delta}_{c}$ to $\bar{C}$ induce the following map:

$$
g: s \bar{C} \stackrel{\Delta_{s} \otimes \bar{\Delta}_{C}}{\longrightarrow} s \mathbb{Q} \otimes s \mathbb{Q} \otimes \bar{C} \otimes \bar{C} \stackrel{\text { id } \otimes \tau \otimes \mathrm{id}}{\longrightarrow} s \mathbb{Q} \otimes \bar{C} \otimes s \mathbb{Q} \otimes \bar{C} .
$$

It induces a degree 1 map $d_{2}$ on $T(s \bar{C})$ satisfying $d_{2}^{2}=0$ because of the coassociativity of $\Delta_{c}$. The two degree 1 maps $d_{1}$ and $d_{2}$ commute (in the graded sense):

$$
d_{1} \circ d_{2}+d_{2} \circ d_{1}=0 .
$$

Lemma 2.13. The complex $\Omega(C)=\left(T(s \bar{C}), d_{\Omega}\right)$ with $d_{\omega}=d_{1}+d_{2}$ and endowed with the concatenation product is an augmented dga algebra called the cobar construction of $C$.

Note that the cobar construction is not in general a quasi-isomorphisms invariant. The reader may look at LV12, Section 2.4] for more details.

2.2.3. Adjunction. The two functors bar and cobar induces an adjunction described as follows:

Theorem 2.14 ([LV12, Theorem 2.2.9 and Corollary 2.3.4]). For every augmented dga algebra $A$ and every conilpotent dga coalgebra $C$ there exist natural bijections

$$
\operatorname{Hom}_{\text {dga alg }}(\Omega(C), A) \simeq \mathrm{Tw}(C, A) \simeq \operatorname{Hom}_{\text {dga coalg }}(C, B(A)) .
$$

The unit $v: C \longrightarrow B \circ \Omega(C)$ and the counit $\epsilon: \Omega \circ B(A) \longrightarrow$ A are quasiisomorphisms of dga coalgebras and algebras respectively. 
2.3. Bar/cobar adjunction: commutative algebras/Lie Coalgebras. In this section we recall the bar/cobar adjunction in the commutative/coLie case giving a pair of functors:

$$
B_{c o m}:\left\{\begin{array}{c}
\text { diff. } \text { gr. com. } \\
\text { ass. algebras }
\end{array}\right\} \rightleftharpoons\left\{\begin{array}{c}
\text { diff. gr. coLie. } \\
\text { coalgebras }
\end{array}\right\}: \Omega_{c o L} .
$$

The cobar construction in the coLie case is a little more delicate. We will concentrate on this construction. The bar construction in the commutative case, will be presented as the set of indecomposable elements of the associative bar construction. A direct construction can be found in [SW11a]. Other descriptions were given in GJ94, GK94.

2.3.1. Cobar construction for Lie coalgebras. The construction follows follows the lines of the commutative dga coalgebra case. However the lack of associativity and the use of the symmetric algebra need to be taken into account.

First we need a notion of conilpotency for a Lie coalgebra $\left(\mathcal{L}^{c}, \delta, d\right)$. As $\delta$ is not associative, one can not directly use an iterated coproduct. One introduces trivalent trees controlling this lack of associativity.

A rooted trivalent tree, or simply a tree, is a planar tree (at each internal vertex a cyclic ordering of the incident edges is given) where vertices have valency 1 (external vertices) or 3 (internal vertices) together with a distinguished external vertex (the root); other external vertices are called leaves. The leaves are numbered from left to right begin at 1 . The trees are drawn with the root (with number 0 ) at the top.

Let $\left(\mathcal{L}^{c}, \delta, d\right)$ be a dg Lie coalgebra. Recall that $\delta$ is a $\operatorname{dg}$ morphism $\delta: \mathcal{L}^{c} \longrightarrow$ $\mathcal{L}^{c} \otimes \mathcal{L}^{c}$

Definition 2.15. Let $T$ be a tree with $n$ as above and let $\left\{e_{1}, \ldots, e_{n}\right\}$ be the set of its leaves ( $e_{i}$ is the $i$-th leaf). $T$ induces a morphism

$$
\delta_{T}: \mathcal{L}^{c} \longrightarrow \mathcal{L}^{c \otimes n}
$$

as follows:

- if $T$ has $n=1$ leaf, $\delta_{T}=$ id $\mathcal{L}^{c}$;

- if $T$ has $n=2$ leaves, then $T=\overbrace{\dot{e}_{1} e_{2}}^{\ominus}$ and $\delta_{T}=\delta$;

- if $T$ has $n \geqslant 3$ leaves, then there exists at least one leaf $e_{i}$ in a strict subtree of the form

$$
T_{0}=\overbrace{\dot{e}_{i}}^{v}{\grave{e_{i+1}}}^{v}
$$

where $v$ is an internal vertex of $T$. Let $T^{\prime}$ be the tree $T \backslash T_{0}$, where the internal vertex $v$ of $T$ is the $i$-th leaf (in $T^{\prime}$ ). The morphism $\delta_{T}$ is defined by

$$
\delta_{T}=\left(\mathrm{id}^{\otimes(i-1)} \otimes \delta \otimes \mathrm{id}^{\otimes(n-i)}\right) \circ \delta_{T^{\prime}} .
$$

This definition does not depend on the choice of the subtree $T_{0}$. By analogy with the associative case, we define.

Definition 2.16. A Lie coalgebra $\left(\mathcal{L}^{c}, \delta, d\right)$ is conilpotent if for any $x \in \mathcal{L}^{c}$ there exist $n$ big enough such that for any tree $T$ with $k$ leaves, $k \geqslant n, \delta_{T}(x)=0$.

We now fix a conilpotent $\mathrm{dg}$ Lie coalgebra $\left(\mathcal{L}^{c}, \delta, d_{\mathcal{L}^{c}}\right)$. Its cobar construction is given by twisting the differential of the free commutative dga $S^{g r}\left(s \mathcal{L}^{c}\right)$. 

by

The differential $d_{\mathcal{L}^{c}}$ induces a differential $d_{s \mathcal{L}^{c}}$ on $s \mathcal{L}^{c}$ and thus on $\left(s \mathcal{L}^{c}\right)^{\otimes n}$ given

$$
\sum_{i=1}^{n} i d^{\otimes(i-1)} \otimes d_{s \mathcal{L}^{c}} \otimes \mathrm{id}^{\otimes(n-i)}:\left(s \mathcal{L}^{c}\right)^{\otimes n} \longrightarrow\left(s \mathcal{L}^{c}\right)^{\otimes n} .
$$

This differential goes down to a differential on $n$-th symmetric power of $s \mathcal{L}^{c}$ :

$$
D_{1}: S^{g r, n}\left(s \mathcal{L}^{c}\right) \longrightarrow S^{g r, n}\left(s \mathcal{L}^{c}\right) .
$$

Using the map $\Delta_{s}$ and the cobracket $\delta_{\mathcal{L}^{c}}$, one has a morphism:

$$
g_{L}: s \mathcal{L}^{c} \stackrel{\Delta_{s} \otimes \mathcal{L}^{c}}{\longrightarrow} s \mathbb{Q} \otimes s \mathbb{Q} \otimes \mathcal{L}^{c} \otimes \mathcal{L}^{c} \stackrel{\text { id } \otimes \tau \otimes i d}{\longrightarrow} s \mathbb{Q} \otimes \mathcal{L}^{c} \otimes s \mathbb{Q} \otimes \mathcal{L}^{c} .
$$

which induces a degree 1 map $\delta^{s}: s \mathcal{L}^{c} \longrightarrow S^{2, g r}\left(s \mathcal{L}^{c}\right)$ because of the relation $\tau \circ \delta=-\delta$ and the sift in the degree.

The relation (id $\left.+\xi+\xi^{2}\right) \circ(\delta \otimes \mathrm{id}) \circ \delta=0$, combined with the shift in the degree and $\Delta_{s}$, shows that $g_{L}$ induce a differential $D_{2}$ on $S^{g r}\left(s \mathcal{L}^{c}\right)$ given by the formula:

$$
\left.D_{2}\right|_{S^{g r, n}\left(s \mathcal{L}^{c}\right)}=\sum_{i=1}^{n} \mathrm{id}^{\otimes(i-1)} \otimes g_{L} \otimes \mathrm{id}^{\otimes(n-i)} .
$$

This is the classical duality between Jacobi identity and $D^{2}=0$ for a classical Lie coalgebra (that is with a dg structure concentrated in degree 0 ).

The differential $D_{1}$ and $D_{2}$ commute (in the graded sens), that is

$$
D_{1} \circ D_{2}+D_{2} \circ D_{1}=0
$$

and one obtains the following.

Lemma 2.17. The complex $\Omega_{c o L}\left(\mathcal{L}^{c}\right)=\left(S^{g r}\left(s \mathcal{L}^{c}\right), d_{\Omega, c o L}\right)$ with the symmetric concatenation product is an augmented commutative dga algebra called the cobarcoLie construction of $\mathcal{L}^{c}$.

2.3.2. Bar construction for commutative dga algebras. Let $\left(A, d_{A}, \mu_{A}, \varepsilon_{A}\right)$ be an augmented commutative dga algebra and $\bar{A}=\operatorname{ker}\left(\varepsilon_{A}\right)$. One can consider its bar construction $B(A)$ as associative algebra. One defines on the coalgebra $B(A)$ an associative product $\amalg$ by the formula

$$
\begin{aligned}
{\left[x_{1}|\cdots| x_{n}\right] \amalg\left[x_{n+1}|\cdots| x_{n+m}\right] } & =\sum_{\sigma \in \operatorname{sh}(n, m)} \rho_{S}(\sigma)\left(\left[x_{1}|\cdots| x_{n}\left|x_{n+1}\right| \cdots \mid x_{n+m}\right]\right) \\
& =\sum_{\sigma \in \operatorname{sh}(n, m)} \varepsilon^{g r}(\sigma)\left(\left[x_{\sigma^{-1}(1)}|\cdots| x_{\sigma^{-1}(n+m)}\right]\right)
\end{aligned}
$$

where $\operatorname{sh}(n, m)$ denotes the subset $\mathbb{S}_{n+m}$ preserving the order of the ordered sets $\{1, \ldots, n\}$ and $\{n+1, \ldots, n+m\}$. For grading reasons, and thus for signs issues, it is important to note that, in the above formula, the $x_{i}$ 's are elements of $s^{-1} A$.

A direct computation shows that $m$ turns $B(A)$ into an augmented commutative dga Hopf algebra; that is it respects the expected diagrams for a Hopf algebra, all the morphisms involved being dg-morphisms.

The augmentation of $B(A)$ is the projection onto the tensor degree 0 part. Let $\overline{B(A)}$ be the kernel of the augmentation of $B(A)$ and

$$
Q_{B}(A)=\overline{B(A)} /(\overline{(B(A)})^{2}
$$

be the set of its indecomposable elements. Ree's theorem [LV12, Theorem 1.3.9] (originally in [Ree58]) shows the following

Lemma 2.18. The differential $d_{B}$ induces a differential $d_{Q}$ on $Q_{B}(A)$. The reduced coproduct $\Delta^{\prime}$ induces a cobracket $\delta_{Q}=1 / 2(\bar{\Delta}-\tau \bar{\Delta})$ on $Q_{B}(A)$ making it into a conilpotent Lie dg coalgebra. 
The complex $\left(Q_{B}(A), d_{Q}\right)$ endowed with the cobracket $\delta_{Q}$ is the commutative bar construction of $A$ and denoted $B_{\text {com }}(A)$.

Working with the indecomposable elements as a quotient may be complicated. In particular, some structure, say for example extra filtrations, may not behave well by taking a quotient. For this purpose, R. Hain, dealing with Hodge structure problems, gave in Hai86 a splitting

$$
i_{Q}: Q_{B}(A) \longrightarrow \overline{B(A)}
$$

of the projection $p_{Q}: \overline{B(A)} \longrightarrow Q_{B}(A)$ commuting with the differential. The projector of $\overline{B(A)}$ given by the composition $p_{\text {шा }}=i_{Q} \circ p_{Q}$ can be express using the following explicit formula given in [Hai86]:

$$
p_{\text {II }}\left(\left[a_{1}|\cdots| a_{n}\right]\right)=\sum_{i=1}^{n} \frac{(-1)^{i-1}}{i} \amalg \circ(\bar{\Delta})^{i-1}\left(\left[a_{1}|\cdots| a_{n}\right]\right)
$$

where the associative product $\amalg$ has been extend to $B(A)^{\otimes n}$ for all $n \geqslant 2$ and where $\bar{\Delta}^{(0)}=$ id .

2.3.3. Adjunction. As in the case of associative algebras and coalgebras, the functors $\Omega_{c o L}$ and $B_{c o m}$ are adjoint.

Theorem 2.19. For any augmented commutative dga algebra $A$ and any conilpotent $d g$ Lie coalgebra $\mathcal{L}^{c}$ there exist natural bijections

$$
\operatorname{Hom}_{\text {com dga alg }}\left(\Omega_{c o L}\left(\mathcal{L}^{c}\right), A\right) \simeq \operatorname{Hom}_{\text {conil dg coLie }}\left(\mathcal{L}^{c}, B_{\text {com }}(A)\right) .
$$

The unit $v: \mathcal{L}^{c} \longrightarrow B_{\text {com }} \circ \Omega_{\text {coL }}\left(\mathcal{L}^{c}\right)$ and the counit $\epsilon: \Omega_{\text {coL }} \circ B_{\text {com }}(A) \longrightarrow A$ are quasi-isomorphisms of conilpotent $d g$ co Lie algebras and commutative dga algebras respectively.

Note that the following diagram of dg vector spaces is commutative:

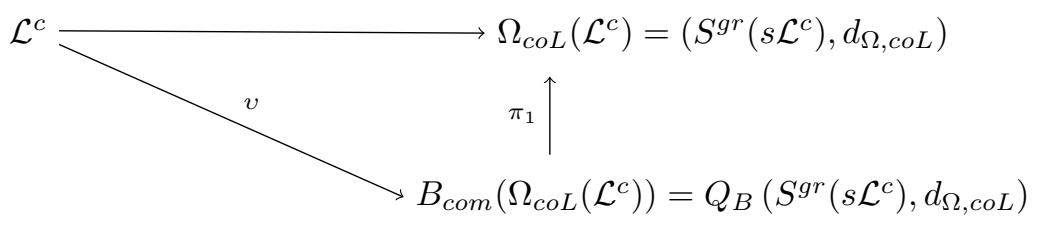

where $\pi_{1}$ is the projection onto the tensor degree 1 part restricted to the set of indecomposable elements; that is restricted to the image of $p_{\mathrm{II}}$.

2.4. An explicit map. We present here an explicit description for the unit $v$ : $\mathcal{L}^{c} \longrightarrow B_{\text {com }} \circ \Omega_{c o L}\left(\mathcal{L}^{c}\right)$ with image in $T^{c}\left(S^{g r}\left(s \mathcal{L}^{c}\right)\right)$ using the projector $p_{\text {II }}$ onto the indecomposable elements.

Definition 2.20. Let $n$ be a positive integer.

- The Catalan number $C(n-1)$ gives the number of rooted trivalent trees with $n$ leaves.

- Let $T$ be such a rooted trivalent tree with $n$ leaves. Define $\tilde{\delta}_{T}$ by

$$
\tilde{\delta}_{T}=\frac{1}{2^{n}} \delta_{T}
$$

where the map $\delta_{T}: \mathcal{L}^{c} \longrightarrow \mathcal{L}^{c \otimes n}$ was introduced at Definition 2.15. 
- The morphism $\tilde{\delta}_{n}$ is up to a normalizing coefficient the sums for all trees $T$ with $n$ leaves of the morphism $\tilde{\Delta}_{T}$ :

$$
\tilde{\Delta}_{n}=\sum_{T} \frac{1}{n C(n-1)} \tilde{\Delta}_{T}
$$

where the sums runs through all trivalent tree with $|T|=n$ leaves.

Note that using the identification $\mathcal{L}^{c} \simeq s^{-1} \otimes s \otimes \mathcal{L}^{c}$ given by $x \mapsto s^{-1} \otimes s \otimes x$, the morphism $\tilde{\Delta}_{n}$ induces a morphism

$$
\mathcal{L}^{c} \longrightarrow\left(s^{-1} \otimes s \otimes \mathcal{L}^{c}\right)^{\otimes n}
$$

again denoted by $\tilde{\Delta}_{n}$.

Claim. The composition

$$
\phi_{\mathcal{L}^{c}}=p_{\text {III }} \circ\left(\sum_{n \geqslant 1} \tilde{\Delta_{n}}\right)
$$

gives a morphism

$$
\phi_{\mathcal{L}^{c}}: \mathcal{L}^{c} \longrightarrow B_{c o m} \circ \Omega_{c o L}\left(\mathcal{L}^{c}\right)=Q_{B}\left(S^{g r}\left(s \mathcal{L}^{c}\right)\right)
$$

which is equal to the unit of the adjunction:

$$
\phi_{\mathcal{L}^{c}}=v .
$$

In the above identification $\Omega_{c o L}\left(\mathcal{L}^{c}\right)=Q_{B}\left(S^{g r}\left(s \mathcal{L}^{c}\right)\right)$, the differential on $\left(S^{g r}\left(s \mathcal{L}^{c}\right)\right)$ is the bar differential $\left.d_{\Omega, c o L}\right)$.

The idea for this formula comes from mimicking the associative case where the unit morphism being a coalgebra morphisms has to be compatible with the iterated reduced coproduct.This formula can be derived as a consequence of the work of Getzler and Jones [GJ94] or of the work of Sinha and Walter [SW11b].

\section{FAMILIES OF BAR ELEMENTS}

In [Sou12], the author defined a family of algebraic cycles $\mathcal{L}_{W}^{\varepsilon}$ indexed by couple $(W, \varepsilon)$ where $W$ is a Lyndon word and $\varepsilon$ is in $\{0,1\}$. Note that, all words considered in this work are words in the two letters 0 and 1.

One of the idea underling the construction of the cycles was to follow explicitly a 1-minimal model construction described in DGMS75 with the hope to use the relation between 1-minimal model and bar construction in order to obtain motives over $\mathbb{P}^{1} \backslash\{0,1, \infty\}$ in the sense of Bloch and Kriz BK94.

The construction of the family of cycles provides in fact a differential system for these cycles related to the action of the free Lie algebra $\operatorname{Lie}\left(X_{0}, X_{1}\right)$ on itself by Ihara's special derivations. In this subsection we will associated bar elements to the previously defined algebraic cycles using the unit of the bar/cobar adjunction in the commutative algebra/Lie coalgebra case.

Before dealing with the algebraic cycles situations, we need to recall the combinatorial situation from Sou12] and its relation with Ihara action. This is need to the related the Lie coalgebra situation (dual to Ihara action) with the differential system for algebraic cycles. 
3.1. Lie algebra, special derivations and Lyndon words. We present here the Lyndon brackets basis for the free Lie algebra $\operatorname{Lie}\left(X_{0}, X_{1}\right)$ and its action on itself by special derivations. The Lie bracket of $\operatorname{Lie}\left(X_{0}, X_{1}\right)$ is denoted by [,] as usual.

A Lyndon word in 0 and 1 is a word in 0 and 1 strictly smaller than any of its nonempty proper right factors for the lexicographic order with $0<1$ (for more details, see [Reu93]).

The standard factorization $[W]$ of a Lyndon word $W$ is defined inductively by $[0]=X_{0},[1]=X_{1}$ and otherwise by $[W]=[[U],[V]]$ with $W=U V, U$ and $V$ nontrivial and such that $V$ is minimal.

Example 3.1. Lyndon words in letters $0<1$ in lexicographic order are up to weight 4:

$$
0<0001<001<0011<01<011<0111<1
$$

Their standard factorization is given in weight 12 and 3 by

$$
[0]=X_{0}, \quad[1]=X_{1}, \quad[01]=\left[X_{0}, X_{1}\right], \quad[001]=\left[X_{0},\left[X_{0}, X_{1}\right]\right] \operatorname{and}\left[\left[X_{0}, X_{1}\right], X_{1}\right] .
$$

In weight 4 , one has

$$
[0001]=\left[X_{0},\left[X_{0},\left[X_{0}, X_{1}\right]\right]\right], \quad \text { and } \quad[0111]=\left[\left[\left[X_{0}, X_{1}\right], X_{1}\right], X_{1}\right]
$$

and

$$
[0011]=\left[X_{0},\left[\left[X_{0}, X_{1}\right], X_{1}\right]\right]
$$

The sets of Lyndon brackets $\{[W]\}$, that is Lyndon words in standard factorization, form a basis of $\operatorname{Lie}\left(X_{0}, X_{1}\right)$ ([Reu93, Theorem 5.1]). This basis can then be used to write the Lie bracket:

Definition 3.2. For any Lyndon word $W$, the coefficients $\alpha_{U, V}^{W}$ (with $U<V$ Lyndon words) are defined by:

$$
[[U],[V]]=\sum_{\substack{\text { Lyndon } \\ \text { words }}} \alpha_{U, V}^{W}[W] .
$$

with $U<V$ Lyndon words. The $\alpha$ 's are the structure coefficients of $\operatorname{Lie}\left(X_{0}, X_{1}\right)$.

A derivation of $\operatorname{Lie}\left(X_{0}, X_{1}\right)$ is a linear endomorphism satisfying

$$
D([f, g])=[D(f), g]+[f, D(g)] \quad \forall f, g \in \operatorname{Lie}\left(X_{0}, X_{1}\right) .
$$

Definition 3.3 (Special derivation, Iha90, Iha92). For any $f$ in $\operatorname{Lie}\left(X_{0}, X_{1}\right)$ we define a derivation $D_{f}$ by:

$$
D_{f}\left(X_{0}\right)=0, \quad D_{f}\left(X_{1}\right)=\left[X_{1}, f\right] .
$$

Ihara bracket on $\operatorname{Lie}\left(X_{0}, X_{1}\right)$ is given by

$$
\{f, g\}=[f, g]+D_{f}(g)-D_{g}(f) .
$$

Ihara bracket is simply the bracket of derivation

$$
\left[D_{1}, D_{2}\right]_{\text {Der }}=D_{1} \circ D_{2}-D_{2} \circ D_{1}
$$

restricted to special derivations :

$$
\left[D_{f}, D_{g}\right]_{D e r}=D_{h}, \quad \text { with } h=\{f, g\} .
$$

Let $L_{1}$ and $L_{x}$ be two copies of the vector space $\operatorname{Lie}\left(X_{0}, X_{1}\right)$. The subscript $x$ denotes a formal variable but it can be think as a point $x$ in $\mathbb{A}^{1} . L_{x}$ is endowed with the free bracket [,] of $\operatorname{Lie}\left[X_{0}, X_{1}\right]$ while $L_{1}$ is endowed with Ihara bracket $\{$,$\} . The Lie algebra L_{1}$ acts on $L_{x}$ by special derivations ; which act on $X_{1}$ hence the subscript. If $f$ is an element of $\operatorname{Lie}\left(X_{0}, X_{1}\right)$, we write $f(1)$ its image in $L_{1}$ and $f(x)$ its image in $L_{x}$. 
Definition 3.4 (GOV97]). The semi-direct sum $L_{1 ; x}$ of $L_{x}$ by $L_{1}$ is as a vector spaces the direct sum

$$
L_{1 ; x}=L_{x} \oplus L_{1}
$$

with bracket $\{,\}_{1 ; x}$ given by [, ] on $L_{x}$, by $\{$,$\} on L_{1}$ and by

$$
\{g(1), f(x)\}_{1 ; x}=-\{f(x), g(1)\}_{1 ; x}=D_{g}(f)(x) \quad \forall f, g \in \operatorname{Lie}\left(X_{0}, X_{1}\right)
$$

on cross-terms.

The union of $\{[W](x),[W](1)\}$ for all Lyndon words gives a basis of $L_{1 ; x}$ while a basis of $L_{1 ; x} \wedge L_{1 ; x}$ is given by the union of the following families

$$
\begin{aligned}
& {[U](x) \wedge[V](x) \text { for any Lyndon word } U<V} \\
& {[U](x) \wedge[V](1) \text { for any Lyndon word } U \neq V} \\
& {[U](1) \wedge[V](1) \text { for any Lyndon word } U<V .}
\end{aligned}
$$

Definition 3.5. The structure coefficients $\alpha_{U, V}^{W}, \beta_{U, V}^{W}$ and $\gamma_{U, V}^{W}$ of $L_{1 ; x}$ are given for any Lyndon words $W$ by the family of relations

$$
\begin{aligned}
& \{[U](x),[V](x)\}_{1 ; x}=\sum_{W \in L y n} \alpha_{U, V}^{W}[W](x) . \quad \text { for any Lyndon word } U<V \\
& \{[U](x),[V](1)\}_{1 ; x}=\sum_{W \in L y n} \beta_{U, V}^{W}[W](x) \quad \text { for any Lyndon word } U \neq V \\
& \{[U](1),[V](1)\}_{1 ; x}=\sum_{W \in L y n} \gamma_{U, V}^{W}[W](1) \quad \text { for any Lyndon word } U<V .
\end{aligned}
$$

All coefficients above are integers.

Because $\{,\}_{1 ; x}$ restricted to $L_{x}$ is the usual cobracket on $\operatorname{Lie}\left(X_{0}, X_{1}\right)$, the $\alpha_{U, V}^{W}$ are the $\alpha$ 's of Definition 3.2; Similarly the $\gamma$ 's are the structure coefficients of Ihara bracket.

Special derivations acts on $X_{1}$ and $D_{X_{0}}$ is simply bracketing with $X_{0}$. This and the above remark show:

Lemma 3.6 ([Sou12, Lemma 4.18]). Let $W$ be a Lyndon word of length greater than or equal to 2. Then the following holds for any Lyndon words $U, V$ :

- $\beta_{0, V}^{W}=0$,

- $\beta_{V, 0}^{W}=\alpha_{0, V}^{W}$

- $\beta_{U, 1}=0$,

- $\beta_{1, U}^{W}=\alpha_{U, 1}^{W}$

- $\gamma_{U, V}^{W}=\alpha_{U, V}^{W}+\beta_{U, V}^{W}-\beta_{V, U}^{W}$.

In particular, $\beta_{0,0}^{W}=\beta_{1,1}^{W}=0$. We also have

$$
\alpha_{U, V}^{\varepsilon}=\beta_{U, V}^{\varepsilon}=\gamma_{U, V}^{\varepsilon}=0
$$

for $\varepsilon \in\{0,1\}$.

3.2. The dual setting : a coaction and a Lie coalgebra. The Lie algebra $\operatorname{Lie}\left(X_{0}, X_{1}\right)$ is graded by the number of letters appearing inside a bracket. Hence there is an induced grading on $L_{1 ; x}$. Taking the graded dual of the $L_{1 ; x}$, we obtain a Lie coalgebra $\mathcal{T}_{1 ; x}^{c o L}$.

Definition 3.7. The elements of the dual basis of the Lyndon bracket basis $[W](x)$ of $L_{x}$ are denoted by $T_{W^{*}}(x)$. Similarly, $T_{W^{*}}(1)$ denotes, for a Lyndon Word $W$ the corresponding element in the basis dual to the basis of $L_{1}$ given by the [W](1)'s. 
For $a$ in $\{1, x\}$, the elements $T_{W^{*}}(a)$ can be represented by a linear combination of rooted trivalent tree with leaves decorated by 0 and 1 and root decorated by $a$ (cf. [Sou12, Section 4.3]). This remark explains the notation which is the same as in Sou12.

A basis of $\mathcal{T}_{1 ; x}^{c o L} \wedge \mathcal{T}_{1 ; x}^{c o L}$ is given by the union of the following families:

$$
\begin{aligned}
& T_{U^{*}}(x) \wedge T_{V^{*}}(x) \text { for any Lyndon word } U<V \\
& T_{U^{*}}(x) \wedge T_{V^{*}}(1) \text { for any Lyndon word } U \neq V \\
& T_{U^{*}}(1) \wedge T_{V^{*}}(1) \text { for any Lyndon word } U<V .
\end{aligned}
$$

By duality between $L_{1 ; x}$ and $\mathcal{T}_{1 ; x}^{c o L}$ one has the following:

Proposition 3.8. The bracket $\{,\}_{1 ; x}$ on $L_{1, x}$ induces a cobracket on $\mathcal{T}_{1 ; x}^{c o L}$

$$
d_{c y}: \mathcal{T}_{1 ; x}^{c o L} \longrightarrow \mathcal{T}_{1 ; x}^{c o L} \wedge \mathcal{T}_{1 ; x}^{c o L}
$$

In terms of the above basis one gets

$$
d_{c y}\left(T_{W^{*}}(x)\right)=\sum_{U<V} \alpha_{U, V}^{W} T_{U^{*}}(x) \wedge T_{V^{*}}(x)+\sum_{U, V} \beta_{U, V}^{W} T_{U^{*}}(x) \wedge T_{V^{*}}(1)
$$

and

$$
d_{c y}\left(T_{W^{*}}(1)\right)=\sum_{U<V} \gamma_{U, V}^{W} T_{U^{*}}(1) \wedge T_{V^{*}}(1)
$$

where $U$ and $V$ are Lyndon words. The coefficients $\alpha_{U, V}^{W}, \beta_{U, V}^{W}$ and $\gamma_{U, V}^{W}$ are those defined in Equation (3).

Note that one has

$$
d_{c y}\left(T_{0^{*}}(x)\right)=d_{c y}\left(T_{0^{*}}(1)\right)=0 \quad \text { and } \quad d_{c y}\left(T_{1^{*}}(x)\right)=d_{c y}\left(T_{1^{*}}(1)\right)=0 .
$$

In weight 2 one has

$$
d_{c y}\left(T_{01}(x)\right)=T_{0^{*}}(x) \wedge T_{1^{*}}(x)+T_{1^{*}}(x) \wedge T_{0^{*}}(1) .
$$

Because of geometric constraints, one can not use directly the combinatorics of the cobracket $d_{c y}$ in this basis to defined a family of algebraic cycle over $\mathbb{P}^{1} \backslash\{0,1, \infty\}$. One defines for any Lyndon word $W$

$$
T_{W^{*}}^{0}=T_{W^{*}}(x) \quad \text { and } \quad T_{W^{*}}^{1}=T_{W^{*}}(x)-T_{W^{*}}(1) .
$$

The definition of $T_{W^{*}}^{0}$ can be thought as the difference $T_{W^{*}}^{0}=T_{W^{*}}(x)-T_{W^{*}}(0)$ where the element $T_{W^{*}}(0)$ is equal to 0 . The elements $T_{W^{*}}^{0}$ and $T_{W^{*}}^{1}$ form a basis of $\mathcal{T}_{1 ; x}^{c o L}$ when $W$ runs through the set of Lyndon words.

Lemma 3.9 ([Sou12, Lemma 4.32]). In this basis the cobracket $d_{c y}$ is given by

$$
d_{c y}\left(T_{W^{*}}^{0}\right)=\sum_{U<V} a_{U, V}^{W} T_{U^{*}}^{0} \wedge T_{V^{*}}^{0}+\sum_{U, V} b_{U, V}^{W} T_{U^{*}}^{1} \wedge T_{V^{*}}^{0},
$$

and

$$
d_{c y}\left(T_{W^{*}}^{1}\right)=\sum_{U<V} a_{U, V}^{\prime W} T_{U^{*}}^{1} \wedge T_{V^{*}}^{1}+\sum_{U, V} b_{U, V}^{\prime W} T_{U^{*}}^{1} \wedge T_{V^{*}}^{0}
$$

where the coefficients $a$ 's, $b$ 's $a^{\prime}$ 's and $b^{\prime}$ 's are given by

$$
\begin{array}{ll}
a_{U, V}^{W}=\alpha_{U, V}^{W}+\beta_{U, V}^{W}-\beta_{V, U}^{W} & \text { for } U<V \\
b_{U, V}^{W}=\beta_{V, U}^{W} & \text { for any } U, V
\end{array}
$$


and

$$
\begin{array}{ll}
a_{U, V}^{\prime W}=-a_{U, V}^{W} & \text { for } U<V, \\
b_{U, V}^{\prime W}=a_{U, V}^{W}+b_{U, V}^{W} & \text { for } U<V, \\
b^{\prime}{ }_{V, U}^{W}=-a_{U, V}^{W}+b_{V, U}^{W} & \text { for } U<V, \\
b_{U, U}^{\prime W}=b_{U, U}^{W} & \text { for any } U .
\end{array}
$$

From the explicit description of the coaction, Lemma 3.6 gives explicitly some of the coefficients $\alpha$ 's and $\beta$ 's. This translates as

Lemma 3.10 ([Sou12, Lemma 4.33]).

- If $W$ is the Lyndon word 0 or 1 , then :

$$
a_{U, V}^{0}=b_{U, V}^{0}=a_{U, V}^{\prime 0}={b_{U, V}^{\prime}}_{U,}^{0}=0, \quad a_{U, V}^{1}=b_{U, V}^{1}=a_{U, V}^{\prime 1}=b_{U, V}^{\prime 1}=0
$$

for any Lyndon words $U$ and $V$.

- For any Lyndon word $W, U$ and $V$ of length at least 2, one has

$$
a_{0, V}^{W}=a_{0, V}^{\prime W}=0, \quad b_{U, 0}^{\prime W}=b_{U, 0}^{\prime W}=0
$$

and

$$
a_{U, 1}^{W}=a_{U, 1}^{\prime W}=0, \quad b_{1, V}^{W}=b_{1, V}^{\prime W}=0 .
$$

Moreover for $W$ a Lyndon word,

$$
a_{U, V}^{W}=b_{U, V}^{W}=a_{U, V}^{\prime W}=b_{U, V}^{\prime W}=0
$$

as soon as the length of $U$ plus the length of $V$ is not equal to the length of $W$.

From the definition of $a_{U, V}^{W}$ and Lemma 3.6 one sees that $a_{U, V}^{W}=\gamma_{U, V}^{W}$. This and Equations $\mathrm{ED}-T^{0}$ and $\left(\mathrm{ED}-T^{1}\right.$ shows that

$$
\begin{aligned}
d_{c y}\left(T_{W^{*}}(1)\right)=d_{c y}\left(T_{W^{*}}^{0}-T_{W^{*}}^{1}\right) & =\sum_{U<V} a_{U, V}^{W}\left(T_{U^{*}}^{0}-T_{U^{*}}^{1}\right) \wedge\left(T_{V^{*}}^{0}-T_{V^{*}}^{1}\right) \\
& =\sum_{U<V} a_{U, V}^{W}\left(T_{U^{*}}^{0}(1)\right) \wedge\left(T_{V^{*}}^{0}(1)\right)
\end{aligned}
$$

3.3. A differential system for cycles. In this subsection we review the cubical complex of quasi-finite cycles over $X$ computing higher Chow groups of $X$. This complex has a natural cdga structure. M. Levine, in [Lev11], proved that the $\mathrm{H}^{0}$ of its bar construction is the tannakian Hopf algebra of mixed Tate motive over $X$.

The ground field is $\operatorname{Spec}(\mathbb{Q})$. The projective line minus three points $\mathbb{P}^{1} \backslash\{0,1, \infty\}$ will be simply denoted by $X$. A generic smooth quasi-projective variety will be denoted by $Y$.

We define $\square^{1}$ to be $\square^{1}=\mathbb{P}^{1} \backslash\{1\}$ and $\square^{n}$ to be $\left(\square^{1}\right)^{n}$. The standard projective coordinates on $\square^{n}$ is $\left[U_{i}: V_{i}\right]$ on the $i$-th factor; and $u_{i}=U_{i} / V_{i}$ is the corresponding affine coordinate. A face $F$ of codimension $p$ of $\square^{n}$ is given by $u_{i_{k}}=\varepsilon_{k}$ for $k=1, \ldots, p$ and $\varepsilon_{k}$ in $\{0, \infty\}$. Such a face is isomorphic to $\square^{n-p}$. For $\varepsilon=0, \infty$ and $i$ in $\{1, \ldots, n\}$, let $s_{i}^{\varepsilon}$ denote the insertion morphism of a codimension 1 face

$$
s_{i}^{\varepsilon}: \square^{n-1} \longrightarrow \square^{n}
$$

given by the identification

$$
\square^{n-1} \simeq \square^{i-1} \times\{\varepsilon\} \times \square^{n-i} .
$$

Definition 3.11 ([Lev11, Example 4.1.6]). Let $Y$ be an irreducible smooth variety. 
- Let $\mathcal{Z}_{q . f .}^{p}(Y, n)$ denote the free abelian group generated by irreducible closed subvarieties

$$
Z \subset Y \times \square^{n} \times\left(\mathbb{P}^{1} \backslash\{1\}\right)^{p}
$$

such that the restriction of the projection on $Y \times \square^{n}$,

$$
p_{1}: Z \longrightarrow Y \times \square^{n}
$$

is dominant and quasi finite (that is of pure relative dimension 0 ).

- We say that elements of $\mathcal{Z}_{q . f .}^{p}(Y, n)$ are quasi-finite.

Intersection with codimension 1 faces give morphisms

$$
\partial_{i}^{\varepsilon}=\left(s_{i}^{\varepsilon}\right)^{*}: \mathcal{Z}_{q . f .}^{p}(Y, n) \longrightarrow \mathcal{Z}_{q . f .}^{p}(Y, n-1)
$$

The symmetric group $\mathfrak{S}_{p}$ acts on $\mathcal{Z}_{q . f}^{p} .(Y, n)$ by permutation of the factors of $\left(\mathbb{P}^{1} \backslash\{1\}\right)^{p}$. Let $S y m_{\mathbb{P}^{1} \backslash\{1\}}^{p}$ denotes the projector corresponding to the symmetric representation.

The symmetric group $\mathfrak{S}_{n}$ acts on $\mathcal{Z}_{\text {q.f. }}^{p}(Y, n)$ by permutation of the factor $\square^{1}$, and $(\mathbb{Z} / 2 \mathbb{Z})^{n}$ acts on $\mathcal{Z}_{q . f .}^{p}(Y, n)$ by $u_{i} \mapsto 1 / u_{i}$ on the $\square^{1}$. The sign representation of $\mathfrak{S}_{n}$ extends to a sign representation

$$
G_{n}=(\mathbb{Z} / 2 \mathbb{Z})^{n} \rtimes \mathfrak{S}_{n} \longrightarrow\{1,-1\} .
$$

Let $\operatorname{Alt}_{n} \in \mathbb{Q}\left[G_{n}\right]$ be the corresponding projector.

Definition 3.12. Let $\mathcal{N}_{Y}^{q f, k}(p)$ denote

$$
\mathcal{N}_{Y}^{q f, k}(p)=\operatorname{Sym}_{\mathbb{P}^{1} \backslash\{1\}}^{p} \circ \operatorname{Alt}_{2 p-k}\left(\mathcal{Z}_{q . f .}^{p}(Y, 2 p-k) \otimes \mathbb{Q}\right) .
$$

- The intersection with codimension 1 faces of $\square^{2 p-k}$ induces a differential

$$
\partial_{Y}=\sum_{i=1}^{2 p-k}(-1)^{i-1}\left(\partial_{i}^{0}-\partial_{i}^{\infty}\right)
$$

of degree 1 .

- The complex of quasi finite cycles is defined by

$$
\mathcal{N}_{Y}^{q f, \bullet}=\mathbb{Q} \oplus \bigoplus_{p \geqslant 1} \mathcal{N}_{Y}^{q f, \bullet}(p)
$$

- Concatenation of factors $\square^{n}$ and of factors $\mathbb{P}^{1} \backslash\{1\}$ followed by the pullback by the diagonal gives a product structure to $\mathcal{N}_{X}^{q f}, \bullet$. This product is graded commutative and $\mathcal{N}_{X}^{q f}, \bullet$ is a cdga ([Lev11, Section 4.2]).

Thanks to VSF00, Chapter IV and VI], the cohomology of $\mathcal{N}_{X}^{q f, \bullet}$ agrees with higher Chow groups of $Y$ tensored with $\mathbb{Q}$ (one can also see [Lev11, Lemma 4.2.1]).

In [Sou12], the author defined two weight 1 degree 1 cycles $\mathcal{L}_{1}^{0}$ and $\mathcal{L}_{0}^{1}$ in $\mathcal{N}_{X}^{q f, 1}$ as the image under $S y m_{\mathbb{P}^{1} \backslash\{1\}}^{1} \circ \mathrm{Alt}_{1}$ of the irreducible varieties defined respectively by:

$$
Z_{0} \subset X \times \square^{1} \times\left(\mathbb{P}^{1} \backslash\{1\}\right):(U-V)(A-B)(U-x V)+x(1-x) U V B=0
$$

and

$$
Z_{1}:(U-V)(A-B)(U-(1-x) V)+x(1-x) U V B=0 .
$$

Starting with these two cycles, the author built in Sou12 two families of degree 1 elements in $\mathcal{N}_{X}^{q f,} \bullet$ whose differential are given by the cobracket in $\mathcal{T}_{1 ; x}^{c o L}$.

Let $j$ be the inclusion $\mathbb{P}^{1} \backslash\{0,1, \infty\}=X \hookrightarrow \mathbb{A}^{1}$. The differential on $\mathcal{N}_{X}^{q f, \bullet}$ is simply denoted by $\partial$.

Theorem 3.13 ([Sou12]). For any Lyndon word of length $p \geqslant 2$, there exist two cycles $\mathcal{L}_{W}^{0}$ and $\mathcal{L}_{W}^{1}$ in $\mathcal{N}_{X}^{q f, 1}(p)$ such that: 
- There exist cycles $\overline{\mathcal{L}_{W}^{0}}, \overline{\mathcal{L}_{W}^{1}}$ in $\mathcal{N}_{\mathbb{A}^{1}}^{q f, 1}(p)$ such that

$$
\mathcal{L}_{W}^{0}=j^{*}\left(\overline{\mathcal{L}_{W}^{0}}\right) \quad \text { and } \quad \mathcal{L}_{W}^{1}=j^{*}\left(\overline{\mathcal{L}_{W}^{1}}\right) .
$$

- The restriction of $\overline{\mathcal{L}_{W}^{0}}$ (resp. $\overline{\mathcal{L}^{1}}$ ) to the fiber $t=0$ (resp. $t=1$ ) is empty.

- The cycle $\mathcal{L}_{W}^{0}$ and $\mathcal{L}_{W}^{1}$ satisfy the following differential equations in $\mathcal{N}_{X}^{q f}$ :

$$
\partial\left(\mathcal{L}_{W}^{0}\right)=-\left(\sum_{U<V} a_{U, V}^{W} \mathcal{L}_{U}^{0} \mathcal{L}_{V}^{0}+\sum_{U, V} b_{U, V}^{W} \mathcal{L}_{U}^{1} \mathcal{L}_{V}^{0}\right)
$$

and

$$
\partial\left(\mathcal{L}_{W}^{1}\right)=-\left(\sum_{U<V} a^{\prime}{ }_{U, V}^{W} \mathcal{L}_{U}^{1} \mathcal{L}_{V}^{1}+\sum_{U, V} b^{\prime}{ }_{U, V}^{W} \mathcal{L}_{U}^{1} \mathcal{L}_{V}^{0}\right)
$$

where coefficients a's, $b$ 's, $a^{\prime}$ 's and $b^{\prime}$ 's are the ones of equations (ED-T $T^{0}$ and ED-T $T^{0}$.

One will write generically a cycle in the above families as $\mathcal{L}_{W}^{\varepsilon}$ with $\varepsilon$ in $\{0,1\}$ when working over $X=\mathbb{P}^{1} \backslash\{0,1, \infty\}$ and $\overline{\mathcal{L}_{W}^{\varepsilon}}$ when working over $\mathbb{A}^{1}$.

The above equations differ from the cobracket in $\mathcal{T}_{1 ; x}^{c o L}$ given at equations $\mathrm{ED}-T^{0}$ and $\left(\mathrm{ED}-T^{1}\right.$ by a global minus sign. This is due to a shift in the degree. Hence the above cycles $\mathcal{L}_{W}^{\varepsilon}$ differ from the ones defined in Sou12] by a global minus sign.

Remark 3.14. The extension $\overline{\mathcal{L}_{W}^{\varepsilon}}$ of the cycles to $\mathcal{N}_{\mathbb{A}^{1}}^{q f} \bullet \bullet$ satisfy the same differential equations as $\mathcal{L}_{W}^{\varepsilon}$ by considering the Zariski closure of the product in the R.H.S. of (ED- $\mathcal{L}^{0}$ and (ED- $\left.\mathcal{L}^{1}\right)$. However, this Zariski closure is not decomposable: terms of the form $\overline{\mathcal{L}_{0}^{1} \mathcal{L}_{V}^{0}}$ are not product in $\mathcal{N}_{\mathbb{A}^{1}}^{q f} \bullet$ because $\overline{\mathcal{L}_{0}^{1}}$ is not in $\mathcal{N}_{\mathbb{A}^{1}}^{q f, 1}$; it is not quasi-finite over 0 (cf proof of Proposition 6.3 in [Sou12]).

Despite the above remark, Theorem 3.13 and the proof of Theorem 5.8 in Sou12 give two other but related families of cycles with decomposable boundary in $\mathcal{N}_{\mathbb{A}^{1}}^{q f, 1}$. Their are described below.

Let $W$ be a Lyndon word of length greater than 2 . We define $\overline{\mathcal{L}_{W}^{0-1}}$ to be the difference

$$
\overline{\mathcal{L}_{W}^{0-1}}=\overline{\mathcal{L}_{W}^{0}}-\overline{\mathcal{L}_{W}^{1}} .
$$

The geometric situation relates $\mathbb{P}^{1} \backslash\{0,1, \infty\}, \mathbb{A}^{1}$ and the point $\{1\}$ as follows:

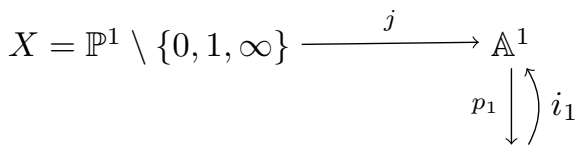

where $j$ is the open inclusion, $p_{1}$ is the projection onto $\{1\}$ and $i_{1}$ the closed inclusion (or the 1-section). We define the constant cycle $\overline{\mathcal{L}_{W}^{0}(1)}$ as

$$
\overline{\mathcal{L}_{W}^{0}(1)}=p_{1}^{*} \circ i_{1}^{*}\left(\overline{\mathcal{L}_{W}^{0}}\right)=p_{1}^{*}\left(\left.\overline{\mathcal{L}_{W}^{0}}\right|_{x=1}\right)
$$

where $\left.\overline{\mathcal{L}_{W}^{0}}\right|_{x=1}$ denotes the fiber at 1 of the cycle $\overline{\mathcal{L}_{W}^{0}}$. Its restriction to $X$ is denote by $\mathcal{L}_{W}^{0}(1)$.

Lemma 3.15. For any Lyndon word of length $p \geqslant 2$ the cycle $\overline{\mathcal{L}_{W}^{0-1}}$ satisfies

$$
\partial\left(\overline{\mathcal{L}_{W}^{0}}-\overline{\mathcal{L}_{W}^{1}}\right)=-\left(\sum_{0<U<V<1} a_{U, V}^{W}\left(\overline{\mathcal{L}_{U}^{0}}-\overline{\mathcal{L}_{U}^{1}}\right)\left(\overline{\mathcal{L}_{V}^{0}}-\overline{\mathcal{L}_{V}^{1}}\right)\right) .
$$


The differential of $\overline{\mathcal{L}_{W}^{0}(1)}$ is given by

$$
\partial\left(\overline{\mathcal{L}_{W}^{0}(1)}\right)=-\left(\sum_{0<U<V<1} a_{U, V}^{W} \overline{\mathcal{L}_{U}^{0}(1) \mathcal{L}_{V}^{0}(1)}\right)
$$

The above equation also holds for $\mathcal{L}_{W}^{0}(1)$ and $i_{1}^{*}\left(\overline{\mathcal{L}_{W}^{0}}\right)$.

Proof. The combinatoric being the same as in $\mathcal{T}_{1 ; x}^{c o L}$, the first part follows from Equation (7). The second part is a consequence of Lemma 3.10 because product of the form $\overline{\mathcal{L}_{U}^{1} \mathcal{L}_{V}^{0}}$ have empty fiber at 1 .

The rest of this section shows that each family of "differential system" gives rise to a family of elements in the corresponding bar constructions.

Let $B_{X}, B_{\mathbb{A}^{1}}$ and $B_{\{1\}}$ denote the bar construction over $\mathcal{N}_{X}^{q f, \bullet}, \mathcal{N}_{\mathbb{A}^{1}}^{q f, \bullet}$ and $\mathcal{N}_{\text {Spec }(\mathbb{Q})}^{q f, \bullet}$ respectively. Let $Q_{X}, Q_{\mathbb{A}^{1}}$ and $Q_{\{1\}}$ be the corresponding set of indecomposable elements. By an abuse of notation, we will write $d_{B}, \Delta$, $ш$ and $\delta_{Q}$ the natural operation in the corresponding spaces. When required by the context, we will precise the "base" space by the subscript $X, \mathbb{A}^{1}$ and $\{1\}$ respectively.

Note that the geometric relation between $X=\mathbb{P}^{1} \backslash\{0,1, \infty\}, A^{1}$ and $\{1\}$ gives rise to morphisms of cdga between the corresponding cycles algebras:

$$
\begin{array}{ll}
\mathcal{N}_{X}^{q f,} \bullet & \mathcal{N}_{\mathbb{A}^{1}}^{q f, \bullet} \\
& \mathcal{N}_{\operatorname{Spec}(\mathbb{Q})}^{q f, \bullet}
\end{array}
$$

which then induce morphisms between bar construction and sets of indecomposable elements. These morphisms are also denoted $j^{*}, p_{1}^{*}$ and $i_{1}^{*}$.

Theorem 3.16 (bar elements). For any Lyndon word $W$ of length $p$ there exist an element $\mathcal{L}_{W}^{B}$, in the bar construction $B_{X}$ satisfying:

- Its image under the projection onto the tensor degree 1 part $\pi_{1}: B_{X} \longrightarrow$ $\mathcal{N}_{X}^{q f}, \bullet$ is $\pi_{1}\left(\mathcal{L}_{W}^{B}\right)=\mathcal{L}_{W}^{0}$.

- Its is in the image of the projector $p_{\mathrm{m}}$; hence it is in $Q_{X}$.

- It is of bar degree 0 and its image under $d_{B}$ is 0 . Thus it induced a class in $\mathrm{H}^{0}\left(B_{X}\right)$ and in $\mathrm{H}^{0}\left(Q_{X}\right)=Q_{\mathrm{H}^{0}\left(B_{X}\right)}$.

- Its image under $\delta_{Q}$ is given by the differential equations (ED- $\mathcal{L}^{0}$ without the minus sign

$$
\delta_{Q}\left(\mathcal{L}_{W}^{0}\right)=\sum_{U<V} a_{U, V}^{W} \mathcal{L}_{U}^{0} \mathcal{L}_{V}^{0}+\sum_{U, V} b_{U, V}^{W} \mathcal{L}_{U}^{1} \mathcal{L}_{V}^{0}
$$

A similar statement holds for $\mathcal{L}_{W}^{1}, \mathcal{L}_{W}^{0-1}$ and $\mathcal{L}_{W}^{0}(1)$ with replace Equation ED- $\mathcal{L}^{0}$ by equation (ED- $\left.\mathcal{L}^{1}\right)$, $\left(\mathrm{ED}-\overline{\mathcal{L}^{0-1}}\right.$ ) and (9).

Proof. The main point is the relation between $\mathcal{T}_{1 ; x}^{c o L}$ and the above family of algebraic cycles and to use the unit of the adjunction cobar/bar.

As in Section 2.3.1, $\Omega_{c o L}\left(\mathcal{T}_{1 ; x}^{c o L}\right)=S^{g r}\left(s \mathcal{T}_{1 ; x}^{c o L}\right)$ denotes the cobar construction over the Lie coalgebra $\mathcal{T}_{1 ; x}^{c o L}$ concentrated purely in degree 0 (hence with 0 as differential). Let

be the morphism of cdga induced by

$$
\psi: \Omega_{c o L}\left(\mathcal{T}_{1 ; x}^{c o L}\right) \longrightarrow \mathcal{N}_{X}^{q f}, \bullet
$$

$$
s T_{W^{*}}^{0} \longmapsto \mathcal{L}_{W}^{0}, \quad s T_{W^{*}}^{1} \longmapsto \mathcal{L}_{W}^{1}
$$


for any Lyndon word $W$ of length $p \geqslant 2$ together with

$$
\psi\left(s T_{0^{*}}^{1}\right)=\mathcal{L}_{1}^{0}, \quad \psi\left(s T_{1^{*}}^{0}\right)=\mathcal{L}_{0}^{1} \text { and } \psi\left(s T_{0^{*}}^{0}\right)=\psi\left(s T_{1^{*}}^{1}\right)=0
$$

where the prefix $s$ denotes the suspension.

The morphism $\psi$ is compatible with the differential because of equations $\mathrm{ED}-\mathcal{L}^{0}$ and $\left(\mathrm{ED}-\mathcal{L}^{1}\right)$ and Lemma 3.10 . The minus sign difference between equations $\mathrm{ED}-\mathcal{L}^{0}$ and (ED-T $T^{0}$ (and similarly for Equation (ED- $\left.\mathcal{L}^{1}\right)$ ) makes it possible to define $\psi$ without sign (cf Section 2.3.1)

It induces a morphism on the bar construction (for the associative case)

$$
\psi_{B}: B\left(\Omega_{c o L}\left(\mathcal{T}_{1 ; x}^{c o L}\right)\right) \longrightarrow B_{X}=B\left(\mathcal{N}_{X}^{q f}, \bullet\right)
$$

compatible with projection on tensor degree $n$ part (for any $n$ ) and with the projector $p_{\text {III }}$ onto the indecomposable elements.

Hence we obtain the following commutative diagram (of vector space)

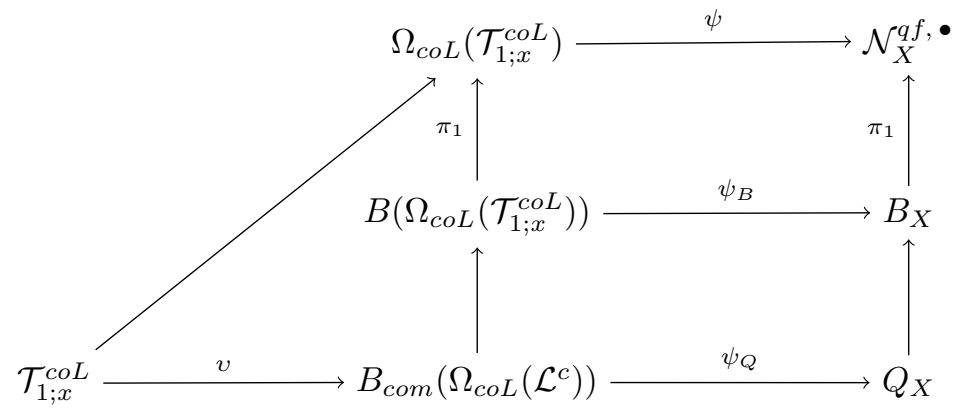

where the morphisms in the bottom line are morphisms of $\mathrm{dg}$ Lie algebras and where $v$ is the unit of the bar/cobar adjunction.

The bar element $\mathcal{L}_{W}^{B}$ is then defined by

$$
\mathcal{L}_{W}^{B}=\psi_{Q} \circ v\left(T_{W^{*}}^{0}\right) .
$$

Similarly we define $\mathcal{L}_{W}^{1, B}$ and $\overline{\mathcal{L}_{W}^{0-1, B}}$.

In order to defined $\mathcal{L}_{W}^{B}(1)$, one consider only the sub Lie coalgebra $\mathcal{T}_{1}^{c o L}$ of $\mathcal{T}_{1 ; x}^{c o L}$ and the morphism

$$
\psi: s T_{W^{*}}(1) \longmapsto \mathcal{L}_{W}^{0}(1)
$$

when $W$ has length $p \geqslant 2$ and sending $s T_{0^{*}}(1)$ and $s T_{1^{*}}(1)$ to zero.

Over $\mathbb{A}^{1}$ a similar statement holds:

Proposition 3.17. For any Lyndon word $W$ of length $p \geqslant 2$, there exists an element $\overline{\mathcal{L}_{W}^{0-1, B}}$ in the bar construction $B_{\mathbb{A}^{1}}$ satisfying

- $\pi_{1}\left(\overline{\mathcal{L}_{W}^{0-1, B}}\right)=\overline{\mathcal{L}_{W}^{0-1}}=\overline{\mathcal{L}_{W}^{0}}-\overline{\mathcal{L}_{W}^{1}}$.

- It is in the image of the projector $p_{\text {II }}$; hence in $Q_{\mathbb{A}^{1}}$.

- It is of bar degree 0 and their image under $d_{B}$ is 0 ; hence it give a class in $\mathrm{H}^{0}\left(B_{X}\right)$ and in $\mathrm{H}^{0}\left(Q_{X}\right)$.

- Its image under $\delta_{Q}$ is given by Equation $\mathrm{ED}-\overline{\mathcal{L}^{0-1}}$ without the minus sign.

- $j^{*}\left(\overline{\mathcal{L}_{W}^{0-1, B}}\right)=\mathcal{L}_{W}^{B}-\mathcal{L}_{W}^{1, B}$.

A similar statement holds for $\overline{\mathcal{L}_{W}^{0}(1)}$ and $i_{1}^{*}\left(\overline{\mathcal{L}_{W}^{0}}\right)=i_{1}^{*}\left(\overline{\mathcal{L}_{W}^{0}(1)}\right)$ with ED- $\overline{\mathcal{L}^{0-1}}$. replace by (9). The corresponding bar elements are denoted

$$
\overline{\mathcal{L}_{W}^{B}(1)} \quad \text { and } \quad \mathcal{L}_{W, x=1}^{B}
$$

respectively. We have the appropriate compatibilities with $p_{1}^{*}$ and $j^{*}$. 
Proof. The proof goes as in Theorem 3.16 above but using only the sub Lie coalgebra $\mathcal{T}_{1}^{c o L}$ and equations $\left(\mathrm{ED}-\overline{\mathcal{L}^{0-1}}\right.$ ) and (9). The coefficients $a_{U, V}^{W}$ appearing in the differential equation for the cycle are equal to coefficients $\gamma_{U, V}^{W}$ giving the cobracket of the element $T_{W^{*}}(1)$ (cf. Lemma 3.6).

The relations between bar elements over $\{1\}, \mathbb{A}^{1}$ and $X=\mathbb{P}^{1} \backslash\{0,1, \infty\}$ follow because $i_{1}^{*}, p_{1}^{*}$ and $j^{*}$ are morphisms of cdga algebra.

Lemma 3.18. In $B_{\mathbb{Q}}$ the following relation holds :

$$
i_{1}^{*}\left(\overline{\mathcal{L}_{W}^{0-1, B}}\right)=i_{1}^{*}\left(\overline{\mathcal{L}_{W}^{B}(1)}\right)=\mathcal{L}_{W, x=1}^{B} .
$$

Proof. It follows from equations (7) and (8) which holds for the cycle on $\mathbb{A}^{1}$ and because in the cycle setting one has in $\mathcal{N}_{\mathbb{Q}}^{q f, \bullet}$

$$
i_{1}^{*}\left(\mathcal{L}_{W}^{0-1}\right)=i_{1}^{*}\left(\overline{\mathcal{L}_{W}^{0}(1)}\right)=\left.\mathcal{L}_{W}^{B}\right|_{x=1}
$$

for any Lyndon word $W$.

\section{A Relative Basis for mixed TAte motive over $\mathbb{P}^{1} \backslash\{0,1, \infty\}$}

4.1. Relations between bar elements. A motivation for introducing cycles $\mathcal{L}_{W}^{1}$ in [Sou12] was the idea of a correspondence

$$
\mathcal{L}_{W}^{0}-\mathcal{L}_{W}^{0}(1) \leftrightarrow \mathcal{L}_{W}^{1}
$$

In this section, we prove that this relation is an equality in the $\mathrm{H}^{0}$ of the bar construction modulo shuffle products; that is

$$
\mathcal{L}_{W}^{B}-\mathcal{L}_{W}^{B}(1)=\mathcal{L}_{W}^{1, B} \quad \in \mathrm{H}^{0}\left(Q_{X}\right)
$$

A key point in order to build cycles $\mathcal{L}_{W}^{0}$ and $\mathcal{L}_{W}^{1}$ in Sou12 was a pull-back by the multiplication. More precisely, the usual multiplication $\mathbb{A}^{1} \times \mathbb{A}^{1} \longrightarrow \mathbb{A}^{1}$ composed with the isomorphism $\mathbb{A}^{1} \times \square^{1} \simeq \mathbb{A}^{1} \times \mathbb{A}^{1}$ gives a multiplication

$$
m_{0}: \mathbb{A}^{1} \times \square^{1} \longrightarrow \mathbb{A}^{1}
$$

sending $(t, u)$ to $\frac{t}{1-u}$. Twisting $m_{0}$ by $\theta: t \mapsto 1-t$ gives a "twisted multiplication"

$$
m_{1}=(\theta \times \text { id }) \circ m_{0} \circ \theta: \mathbb{A}^{1} \times \square^{1} \longrightarrow \mathbb{A}^{1} .
$$

Proposition 4.1 ([Sou12]). For $\varepsilon=0,1$ the morphism $m_{\varepsilon}$ induces a linear morphism

giving a homotopy

$$
m_{\varepsilon}^{*}: \mathcal{N}_{\mathbb{A}^{1}}^{q f, k} \longrightarrow \mathcal{N}_{\mathbb{A}^{1}}^{q f, k-1}
$$

$$
\partial_{\mathbb{A}^{1}} \circ m_{\varepsilon}^{*}+m_{\varepsilon}^{*} \circ \partial_{\mathbb{A}^{1}}=\mathrm{id}-p_{\varepsilon}^{*} \circ i_{\varepsilon}^{*}
$$

where $p_{\varepsilon}: \mathbb{A}^{1} \longrightarrow\{\varepsilon\}$ is the projection onto the point $\{\varepsilon\}$ and $i_{\varepsilon}$ its inclusion is $\mathbb{A}^{1}$.

From this homotopy property, one derives the following relation between $m_{0}^{*}$ and $m_{1}^{*}$.

Lemma 4.2. One has:

$$
m_{1}^{*}=m_{0}^{*}-p_{1}^{*} \circ i_{1}^{*} \circ m_{0}^{*}-\partial_{\mathbb{A}^{1}} \circ m_{1}^{*} \circ m_{0}^{*}+m_{1}^{*} \circ m_{0}^{*} \circ \partial_{\mathbb{A}^{1}}+m_{1}^{*} \circ p_{0}^{*} \circ i_{0}^{*}
$$

and a similar expression for $m_{1}^{*}$.

In particular, when $b \in \mathcal{N}_{\mathbb{A}^{1}}^{q f, k}$ satisfies $\partial_{\mathbb{A}^{1}}(b)=0$ and $i_{0}(b)=0$, one has:

$$
m_{1}^{*}(b)=m_{0}^{*}(b)-p_{1}^{*} \circ i_{1}^{*}\left(m_{0}^{*}(b)\right)+\partial_{\mathbb{A}^{1}}\left(m_{0}^{*} \circ m_{1}^{*}(b)\right)
$$


Proof. Let $b$ be in $\mathcal{N}_{\mathbb{A}^{1}}^{q f, k}$. We treat only Equation 10, Using the homotopy property for $m_{0}^{*}$, one writes

$$
b=\partial_{\mathbb{A}^{1}} \circ m_{0}^{*}(b)+m_{0}^{*} \circ \partial_{\mathbb{A}^{1}}+p_{0}^{*} \circ i_{0}^{*}(b) .
$$

Computing $m_{1}^{*}(b)$, the homotopy property

$$
m_{1}^{*} \circ \partial_{\mathbb{A}^{1}}\left(m_{0}(b)\right)=m_{0}^{*}(b)-p_{1}^{*} \circ i_{1}^{*}\left(m_{0}(b)\right)-\partial_{\mathbb{A}^{1}} \circ m_{1}^{*}\left(m_{0}^{*}(b)\right)
$$

gives the desired formula.

Writing $\overline{A_{W}}$ the $\mathbb{A}^{1}$-extension of the right hand side of Equation $\left(\mathrm{ED}-\mathcal{L}^{0}\right.$, cycles $\overline{\mathcal{L}_{W}^{0}}$ are obtained in Sou12] as

$$
\overline{\mathcal{L}_{W}^{0}}=m_{0}^{*}\left(\overline{A_{W}}\right)
$$

and similarly for $\mathcal{L}_{W}^{1}$

An explicit computation in low weight Sou12, Sou13 shows that

$$
\overline{\mathcal{L}_{01}^{0}}=m_{0}^{*}\left(\overline{\mathcal{L}_{0}^{0}} \overline{\mathcal{L}_{1}^{0}}\right) \quad \text { and } \quad \overline{\mathcal{L}_{01}^{1}}=m_{1}^{*}\left(\overline{\mathcal{L}_{0}^{0}} \overline{\mathcal{L}_{1}^{0}}\right) .
$$

Using Lemma 4.2, one gets

$$
\overline{\mathcal{L}_{01}^{1}}=\overline{\mathcal{L}_{01}^{0}}-\overline{\mathcal{L}_{01}^{0}(1)}+\partial_{\mathbb{A}^{1}}\left(m_{1}^{*} \circ m_{0}^{*}\left(\overline{\mathcal{L}_{0}^{0}} \overline{\mathcal{L}_{1}^{0}}\right)\right) .
$$

Note that as a parametrized cycle,

$$
m_{1}^{*} \circ m_{0}^{*}\left(\overline{\mathcal{L}_{0}^{0}} \overline{\mathcal{L}_{1}^{0}}\right)=m_{1}^{*}\left(\overline{\mathcal{L}_{01}^{0}}\right)
$$

can be written (omitting the projector Alt) as

$$
m_{1}^{*} \circ m_{0}^{*}\left(\overline{\mathcal{L}_{0}^{0}} \overline{\mathcal{L}_{1}^{0}}\right)=\left[t ; \frac{y-t}{y-1}, 1-\frac{y}{x}, x, 1-x\right] \subset \mathbb{A}^{1} \times \square^{4} .
$$

This expression coincides, up to reparametrization, with the expression of $C_{01}$ given in [Sou13, Example 5.5] relating $\mathcal{L}_{01}^{0}$ and $\mathcal{L}_{01}^{1}$.

Thus, $\overline{\mathcal{L}_{01}^{0}}-\overline{\mathcal{L}_{01}^{0}(1)}$ and $\overline{\mathcal{L}_{01}^{1}}$ differs only by a boundary. The differential of $\overline{\mathcal{L}_{01}^{0}(1)}$ is zero and one can compute explicitly the corresponding bar elements:

$$
\overline{\mathcal{L}_{01}^{0-1, B}}=\left[\mathcal{L}_{01}^{0}\right]-\left[\mathcal{L}_{01}^{1}\right], \quad \mathcal{L}_{01}^{B}(1)=\left[\mathcal{L}_{01}^{0}(1)\right]
$$

Lemma 4.3. In $B\left(\mathcal{N}_{A^{1}}^{q f, \bullet}\right)$, one has the following relation

$$
\overline{\mathcal{L}_{01}^{0-1, B}}-\mathcal{L}_{01}^{B}(1)=d_{B}\left(\left[m_{1}^{*}\left(\overline{\mathcal{L}_{01}^{0}}\right)\right]\right) .
$$

Thus in $\mathrm{H}^{0}\left(B_{\mathbb{A}^{1}}\right)$ as in $\mathrm{H}^{0}\left(Q_{\mathbb{A}^{1}}\right)$ one has the equality between

$$
\overline{\mathcal{L}_{01}^{0-1, B}}-\mathcal{L}_{01}^{B}(1)=0 .
$$

Taking the restriction to $\mathbb{P}^{1} \backslash\{0,1, \infty\}$, one obtains in $\mathrm{H}^{0}\left(Q_{X}\right)$

$$
\mathcal{L}_{01}^{B}-\mathcal{L}_{01}^{1, B}=j^{*}\left(\overline{\mathcal{L}_{01}^{0-1, B}}\right)=\mathcal{L}_{01}^{B}(1) .
$$

For $W$ a Lyndon word of length $p \geqslant 2$, the explicit comparison between $\mathcal{L}_{W}^{B}$ and $\mathcal{L}_{W}^{1, B}$ is in general much more complicated as

$$
\partial_{\mathbb{A}^{1}}\left(\overline{\mathcal{L}_{W}^{0}}-\overline{\mathcal{L}_{W}^{1}}\right)=-\sum_{0<U<V<1} a_{U, V}^{W}\left(\overline{\mathcal{L}_{U}^{0}}-\overline{\mathcal{L}_{U}^{1}}\right)\left(\overline{\mathcal{L}_{V}^{0}}-\overline{\mathcal{L}_{V}^{1}}\right) \neq 0
$$

However, working at the bar construction level in $\mathrm{H}^{0}\left(Q_{\mathbb{A}^{1}}\right)$ allows to use an induction argument. 
Theorem 4.4. For any Lyndon word $W$ of length $p \geqslant 2$ the following relation holds

$$
\overline{\mathcal{L}_{W}^{0-1, B}}=\overline{\mathcal{L}_{W}^{B}(1)} \quad \text { in } \quad \mathrm{H}^{0}\left(Q_{\mathbb{A}^{1}}\right)=Q_{\mathrm{H}^{0}\left(B_{\mathbb{A}^{1}}\right)} .
$$

Taking the restriction to $X=\mathbb{P}^{1} \backslash\{0,1, \infty\}$, one obtains in $\mathrm{H}^{0}\left(Q_{X}\right)=Q_{\mathrm{H}^{0}\left(B_{X}\right)}$

$$
\mathcal{L}_{W}^{B}-\mathcal{L}_{W}^{1, B}=j^{*}\left(\overline{\mathcal{L}_{W}^{0-1, B}}\right)=\mathcal{L}_{W}^{B}(1) .
$$

Proof. From Lemma 4.3 above it is true for $p=2$ as there is then only one Lyndon word to consider $W=01$.

Now we assume that the theorem is true for all Lyndon words of length $k$ with $2 \leqslant k \leqslant p-1$. Let $W$ be a Lyndon word of length $p$.

From Proposition 3.17 , one has in $Q_{\mathbb{A}^{1}}$ and in particular in $\mathrm{H}^{0}\left(Q_{\mathbb{A}^{1}}\right)$ :

$$
\delta_{Q}\left(\overline{\mathcal{L}_{W}^{0-1, B}}\right)=\sum_{0<U<V<1} a_{U, V}^{W}\left(\overline{\mathcal{L}_{U}^{0-1, B}}\right) \wedge\left(\overline{\mathcal{L}_{V}^{0-1, B}}\right)
$$

and

$$
\delta_{Q}\left(\overline{\mathcal{L}_{W}^{B}(1)}\right)=\sum_{0<U<V<1} a_{U, V}^{W}\left(\overline{\mathcal{L}_{U}^{B}(1)}\right) \wedge\left(\overline{\mathcal{L}_{V}^{B}(1)}\right) .
$$

Using the induction hypothesis, one has in $\mathrm{H}^{0}\left(Q_{\mathbb{A}^{1}}\right)$

$$
\delta_{Q}\left(\overline{\mathcal{L}_{W}^{0-1, B}}\right)=\sum_{0<U<V<1} a_{U, V}^{W}\left(\overline{\mathcal{L}_{U}^{B}(1)}\right) \wedge\left(\overline{\left(\mathcal{L}_{V}^{B}(1)\right.}\right)
$$

and thus

$$
\delta_{Q}\left(\overline{\mathcal{L}_{W}^{0-1, B}}-\overline{\mathcal{L}_{W}^{B}(1)}\right)=0 \quad \text { in } \quad \mathrm{H}^{0}\left(Q_{\mathbb{A}^{1}}\right) .
$$

Let $C_{W}$ be the class of $\overline{\mathcal{L}_{W}^{0-1, B}}-\overline{\mathcal{L}_{W}^{B}(1)}$ in $\mathrm{H}^{0}\left(Q_{\mathbb{A}^{1}}\right)$ and $s C_{W}$ its image in

$$
\Omega_{c o L}\left(\mathrm{H}^{0}\left(Q_{\mathbb{A}^{1}}\right)\right)=S^{g r}\left(s \mathbb{Q} \otimes \mathrm{H}^{0}\left(Q_{\mathbb{A}^{1}}\right)\right) .
$$

As $\delta_{Q}\left(C_{W}\right)=0, d_{\Omega, c o L}\left(s C_{W}\right)=0$ and $s C_{W}$ gives a class in

$$
\mathrm{H}^{1}\left(\Omega_{c o L}\left(\mathrm{H}^{0}\left(Q_{A^{1}}\right)\right)\right) \simeq \mathrm{H}^{1}\left(\mathcal{N}_{\mathbb{A}^{1}}^{q f, \bullet}\right)
$$

where the above isomorphism is given by Bloch and Kriz in [BK94, Corollary 2.31] after a choice of a 1-minimal model in the sens of Sullivan. Using the comparison between $\mathrm{H}^{1}\left(\mathcal{N}_{\mathbb{A}^{1}}^{q f, \bullet}\right)$ and the higher Chow groups, this class can be represented by $p_{1}^{*}(C)$ in $\mathcal{N}_{\mathbb{A}^{1}}^{q f, 1}$ with $C$ a cycle in $\mathcal{N}_{\mathbb{Q}}^{q f, 1}$.

The cycle $p_{1}^{*}(C)$ satisfies $\partial_{\mathbb{A}^{1}}\left(p_{1}^{*}(C)\right)=0$ and $\left[p_{1}^{*}(C)\right]$ gives a degree 0 bar element $C^{B}$ in $B_{\mathbb{A}^{1}}$ whose bar differential and reduced coproduct are equal to 0 .

From this, one gets a class $\tilde{C}_{W}=C_{W}-C^{B}$ in $\mathrm{H}^{0}\left(Q_{\mathbb{A}^{1}}\right)$. Its image $s \tilde{C}_{W}$ in $\Omega_{c o L}\left(\mathrm{H}^{0}\left(Q_{A^{1}}\right)\right)$ also gives a class in

$$
\mathrm{H}^{1}\left(\Omega_{c o L}\left(\mathrm{H}^{0}\left(Q_{A^{1}}\right)\right)\right)
$$

which is 0 by construction.

As, on the degree 0 part of $\Omega_{c o L}\left(H^{0}\left(Q_{\mathbb{A}^{1}}\right)\right)$, the differential $d_{\Omega, c o L}$ is zero, one obtains that $s \tilde{C}_{W}=0$ in $s \mathbb{Q} \otimes \mathrm{H}^{0}\left(Q_{A^{1}}\right)$ and thus $\tilde{C}_{W}$ is zero in $\mathrm{H}^{0}\left(Q_{A^{1}}\right)=Q_{\mathrm{H}^{0}\left(B_{\mathbb{A}^{1}}\right)}$. The above discussion shows that:

$$
0=\tilde{C}_{W}=C_{W}-C^{B}=C_{W}-\left[p_{1}^{*}(C)\right]
$$

So far one has obtained that in $B_{\mathbb{A}^{1}}$ :

$$
\overline{\mathcal{L}_{W}^{0-1, B}}-\overline{\mathcal{L}_{W}^{B}(1)}-\left[p_{1}^{*}(C)\right]=d_{B}(b) \quad \text { modulo } ш \text { products }
$$

with $b$ in the degree -1 part of $B_{\mathbb{A}^{1}}=B\left(\mathcal{N}_{\mathbb{A}^{1}}^{q f}, \bullet\right)$. 
Because taking the fiber at 1 commutes with products and differential, one gets modulo shuffles

$$
i_{1}^{*}\left(\overline{\mathcal{L}_{W}^{0-1, B}}\right)-i_{1}^{*}\left(\overline{\mathcal{L}_{W}^{B}(1)}\right)-[C]=d_{B}\left(i_{1}^{*}(b)\right) .
$$

Lemma 3.18 insures that $i_{1}^{*}\left(\overline{\mathcal{L}_{W}^{0-1, B}}\right)-i_{1}^{*}\left(\overline{\mathcal{L}_{W}^{B}(1)}\right)=0$. Thus one has

$$
-[C]=d_{B}\left(i_{1}^{*}(b)\right)+\text { shuffle products }
$$

which shows that $\left[p^{*}(C)\right]$ is zero in $\mathrm{H}^{0}\left(B_{\mathbb{A}^{1}}\right)$ modulo shuffles. Hence Equation (16) can be written has

$$
\overline{\mathcal{L}_{W}^{0-1, B}}-\overline{\mathcal{L}_{W}^{B}(1)}=0 \quad \text { in } \quad Q_{\mathrm{H}^{0}\left(B_{\mathbb{A}^{1}}\right)}=\mathrm{H}^{0}\left(Q_{\mathbb{A}^{1}}\right)
$$

Finally, taking the restriction to $\mathbb{P}^{1} \backslash\{0,1, \infty\}$, one has $\mathcal{L}_{W}^{B}-\mathcal{L}_{W}^{1, B}=j^{*}\left(\overline{\mathcal{L}_{W}^{0-1, B}}\right)$.

The main consequence of Equation (15) in the previous theorem is that in $Q_{\mathrm{H}^{0}\left(B_{X}\right)}$ one can replace the bar avatar of the geometric differential system ED- $\mathcal{L}^{0}$ by a bar avatar of the differential system (ED-T) coming from Ihara action by special derivations.

Corollary 4.5. In $Q_{\mathrm{H}^{0}\left(B_{X}\right)}$, the set of indecomposable elements of $\mathrm{H}^{0}\left(B\left(\mathcal{N}_{X}^{q f}, \bullet\right)\right)$, the following holds for any (non-empty) Lyndon word $W$ :

$$
\delta_{Q}\left(\mathcal{L}_{W}^{B}\right)=\sum_{U<V} \alpha_{U, V}^{W} \mathcal{L}_{U}^{B} \wedge \mathcal{L}_{V}^{B} \sum_{U, V} \beta_{U, V}^{W} \mathcal{L}_{U}^{B} \wedge \mathcal{L}_{V}^{B}(1)
$$

Proof. Let $W$ be a Lyndon word. The statement holds when $W$ has length equal 1 and one can assume that $W$ has length greater or equal to 2 . One begins with the formula giving $\delta_{Q}\left(\mathcal{L}_{W}^{B}\right)$ from Theorem 3.16

$$
\delta_{Q}\left(\mathcal{L}_{W}^{B}\right)=\sum_{U<V} a_{U, V}^{W} \mathcal{L}_{U}^{B} \wedge \mathcal{L}_{V}^{B} \sum_{U, V} b_{U, V}^{W} \mathcal{L}_{U}^{B} \wedge \mathcal{L}_{V}^{1, B}
$$

Then using the relations given by Equation (15), one has

$$
\delta_{Q}\left(\mathcal{L}_{W}^{B}\right)=\sum_{U<V} a_{U, V}^{W} \mathcal{L}_{U}^{B} \wedge \mathcal{L}_{V}^{B} \sum_{U, V} b_{U, V}^{W} \mathcal{L}_{U}^{B} \wedge\left(\mathcal{L}_{V}^{B}-\mathcal{L}_{V}^{B}(1)\right)
$$

Expanding terms as $\mathcal{L}_{U}^{B}\left(\mathcal{L}_{V}^{B}-\mathcal{L}_{V}^{B}(1)\right)$ and we conclude the proof using the expression of coefficients $a$ 's and $b$ ' in terms of $\alpha$ 's and $\beta$ 's given at Lemma 3.9.

4.2. A Basis for the geometric Lie coalgebra. This section shows that the image of the family of bar elements $\mathcal{L}_{W}^{B}$ in Deligne-Goncharov motivic fundamental Lie coalgebra is a basis of this coLie coalgebra. Hence the family $\mathcal{L}_{W}^{B}$ induced a basis of the tannakian coLie coalgebra of mixed Tate motives over $\mathbb{P}^{1} \backslash\{0,1, \infty\}$ relative to the one for mixed Tate motives over $\mathbb{Q}$.

We recall that for $X=\mathbb{P}^{1} \backslash\{0,1, \infty\}$, M. Levine in Lev11] [Theorem 5.3.2 and beginning of the section 6.6] shows, one can identify the Tannakian group associated with $\operatorname{MTM}(X)$ with the spectrum of $\mathrm{H}^{0}\left(B_{X}\right)$ :

$$
G_{\mathrm{MTM}(X)} \simeq \operatorname{Spec}\left(\mathrm{H}^{0}\left(B_{X}\right)\right) .
$$

Then, he uses a relative bar-construction in order to relate $G_{M T M(X)}$ to the motivic fundamental group of $X$ of Goncharov and Deligne, $\pi_{1}^{\operatorname{mot}}(X, x)$ (see [Del89] and [DG05]).

Theorem 4.6 ([Lev11]|Corollary 6.6.2]). Let $x$ be a $\mathbb{Q}$-point of $X=\mathbb{P}^{1} \backslash\{0,1, \infty\}$. Then there is a split exact sequence: 


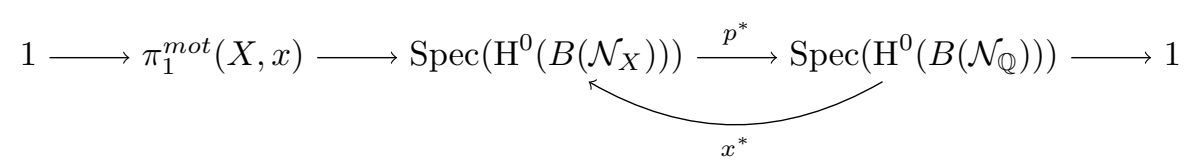

where $p$ is the structural morphism $p: \mathbb{P}^{1} \backslash\{0,1, \infty\} \longrightarrow \operatorname{Spec}(\mathbb{Q})$.

Theorem 4.6 can be reformulate in terms of Lie coalgebras, looking at indecomposable elements of the respective Hopf algebras.

Proposition 4.7. There is a split exact sequence of Lie coalgebras:

$$
0 \longrightarrow Q_{\mathrm{H}^{0}\left(B_{Q}\right)}^{\stackrel{\tilde{p}}{\nwarrow}{ }_{\tilde{x}}^{\longrightarrow}} Q_{\mathrm{H}^{0}\left(B_{X}\right)} \stackrel{\phi}{\longrightarrow} Q_{\text {geom }} \longrightarrow 0
$$

where $Q_{\text {geom }}$ is the set of indecomposable elements of $\mathcal{O}\left(\pi_{1}^{m o t} X, x\right)$ and is isomorphic as Lie coalgebra to the graded dual of the Lie algebra associated to $\pi_{1}^{m o t}(X, x)$. Hence, $Q_{\text {geom }}$ is isomorphic as Lie coalgebra to the graded dual of the free Lie algebra on two generators $\operatorname{Lie}\left(X_{0}, X_{1}\right)$.

Considering the family of bar elements $\mathcal{L}_{W}^{B}$ for all Lyndon words $W$ in this short exact sequence of Lie coalgebra, ones gets

Theorem 4.8. The family $\phi\left(\mathcal{L}_{W}^{B}\right)$ for any Lyndon words $W$ is a basis of the Lie coalgebra $Q_{\text {geom }}$. Hence the family $\mathcal{L}_{W}^{B}$ is a basis of $Q_{\mathrm{H}^{0}\left(B_{X}\right)}$ relatively to $Q_{\mathrm{H}^{0}\left(B_{\mathbb{Q}}\right)}$.

Proof. The above short exact sequence being a sequence of Lie coalgebra, one has

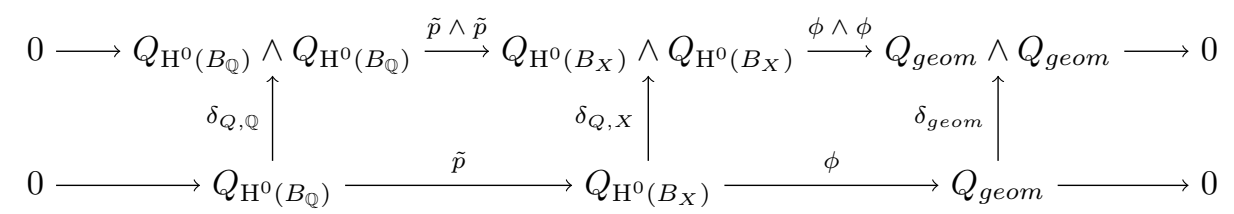

As $\delta_{Q, X}\left(\mathcal{L}_{0}^{B}\right)=\delta_{Q, X}\left(\mathcal{L}_{1}^{B}\right)=0$, weight reasons show that $\phi\left(\mathcal{L}_{0}^{B}\right)$ and $\phi\left(\mathcal{L}_{1}^{B}\right)$ are dual to the weight 1 generators of $\operatorname{Lie}\left(X_{0}, X_{1}\right)$.

In order to show that the family $\phi\left(\mathcal{L}_{W}^{B}\right)$ is a basis of $Q_{g e o m}$, it is enough to show that the elements $\phi\left(\mathcal{L}_{W}^{B}\right)$ satisfy:

$$
\delta_{\text {geom }}\left(\phi\left(\mathcal{L}_{W}^{B}\right)\right)=\sum_{U<V} \alpha_{U, V}^{W} \phi\left(\mathcal{L}_{U}^{B}\right) \wedge \phi\left(\mathcal{L}_{V}^{B}\right)
$$

because $\delta_{\text {geom }}$ is dual to the bracket [, ] of $\operatorname{Lie}\left(X_{0}, X_{1}\right)$.

As $\phi$ commutes with the cobracket, it is enough to compute $(\phi \wedge \phi) \circ \delta_{X}\left(\mathcal{L}_{W}^{B}\right)$ :

$$
\begin{aligned}
\delta_{\text {geom }}\left(\phi\left(\mathcal{L}_{W}^{B}\right)\right) & =(\phi \wedge \phi) \circ \delta_{X}\left(\mathcal{L}_{W}^{B}\right) \\
& =(\phi \wedge \phi)\left(\sum_{U<V} \alpha_{U, V}^{W} \mathcal{L}_{U}^{B} \wedge \mathcal{L}_{V}^{B} \sum_{U, V} \beta_{U, V}^{W} \mathcal{L}_{U}^{B} \wedge \mathcal{L}_{V}^{B}(1)\right) \\
& =\sum_{U<V} \alpha_{U, V}^{W} \phi\left(\mathcal{L}_{U}^{B}\right) \wedge \phi\left(\mathcal{L}_{V}^{B}\right) \sum_{U, V} \beta_{U, V}^{W} \phi\left(\mathcal{L}_{U}^{B}\right) \wedge \phi\left(\mathcal{L}_{V}^{B}(1)\right)
\end{aligned}
$$

By construction $\phi\left(\mathcal{L}_{V}^{B}(1)\right)$ is zero. Thus one obtains the expected formula for $\delta_{\text {geom }}\left(\phi\left(\mathcal{L}_{W}^{B}\right)\right)$. 
Note that $\delta_{X}$ gives the coaction of $Q_{\mathrm{H}^{0}\left(B_{\mathbb{Q}}\right)}$ on $Q_{\text {geom }}$ described in Bro12] in relation with Goncharov motivic coproduct $\Delta^{m o t}$. In this context, Equation (ED- $Q_{X}$

$$
\delta_{Q}\left(\mathcal{L}_{W}^{B}\right)=\sum_{U<V} \alpha_{U, V}^{W} \mathcal{L}_{U}^{B} \wedge \mathcal{L}_{V}^{B} \sum_{U, V} \beta_{U, V}^{W} \mathcal{L}_{U}^{B} \wedge \mathcal{L}_{V}^{B}(1)
$$

is nothing but another expression for Goncharov motivic cobracket $1 / 2\left(\Delta^{\text {mot }}-\right.$ $\left.\tau \Delta^{m o t}\right)$. This new expression has the advantage that it is stable under the generating family $\mathcal{L}_{W}^{B}$.

\section{REFERENCES}

[BK94] Spencer Bloch and Igor Kriz, Mixed tate motives, Anna. of Math. 140 (1994), no. 3, $557-605$

[Bro09] Francis C. S. Brown, Multiple zeta values and periods of moduli spaces $\overline{\mathfrak{M}}_{0, n}$, Ann. Sci. Éc. Norm. Supér. (4) 42 (2009), no. 3, 371-489.

[Bro12] Francis Brown, Mixed Tate motives over $\mathbb{Z}$, Ann. of Math. (2) 175 (2012), no. 2, 949-976. MR 2993755

[Del89] Pierre Deligne, Le groupe fondamental de la droite projective moins trois points, Galois groups over $\mathbb{Q}$, MSRI Publ, vol. 16, Springer Verlag, 1989, pp. 70-313.

[DG05] Pierre Deligne and Alexander B. Goncharov, Groupes fondamentaux motiviques de Tate mixte, Ann. Sci. École Norm. Sup. (4) 38 (2005), no. 1, 1-56.

[DGMS75] Pierre Deligne, Phillip Griffiths, John Morgan, and Dennis Sullivan, Real homotopy theory of Kähler manifolds, Invent. Math. 29 (1975), no. 3, 245-274.

[GGL09] H. Gangl, A. B. Goncharov, and A. Levin, Multiple polylogarithms, polygons, trees and algebraic cycles, Algebraic geometry-Seattle 2005. Part 2, Proc. Sympos. Pure Math., vol. 80, Amer. Math. Soc., Providence, RI, 2009, pp. 547-593.

[GJ94] Ezra Getzler and J. D. S. Jones, Operads, homotopy algebra and iterated integrals for double loop spaces, http://arxiv.org/abs/hep-th/9403055, 1994.

[GK94] Victor Ginzburg and Mikhail Kapranov, Koszul duality for operads, Duke Math. J. 76 (1994), no. 1, 203-272.

[Gon05] A. B. Goncharov, Galois symmetries of fundamental groupoids and noncommutative geometry, Duke Math. J. 128 (2005), no. 2, 209-284.

[GOV97] V. V. Gorbatsevich, A. L. Onishchik, and E. B. Vinberg, Foundations of Lie theory and Lie transformation groups, Springer-Verlag, Berlin, 1997, Translated from the Russian by A. Kozlowski, Reprint of the 1993 translation [1t Lie groups and Lie algebras. I, Encyclopaedia Math. Sci., 20, Springer, Berlin, 1993; MR1306737 (95f:22001)].

[Hai86] Richard M. Hain, On the indecomposable elements of the bar construction, Proc. Amer. Math. Soc. 98 (1986), no. 2, 312-316.

[Iha90] Yasutaka Ihara, Automorphisms of pure sphere braid groups and Galois representations, The Grothendieck Festschrift, Vol. II, Progr. Math., vol. 87, Birkhäuser Boston, Boston, MA, 1990, pp. 353-373.

[Iha92] _ On the stable derivation algebra associated with some braid groups, Israel J. Math 80 (1992), no. 1-2, 135-153.

[Lev93] M. Levine, Tate motives and the vanishing conjecture for algebraic $k$-theory, Algebraic K-Theory and Algebraic Topology, Lake Louise 1991 (Paul G. Goerss and John F. Jardine, eds.), NATO Adv. Sci. Inst. Ser C Math. Phys. Sci., no. 407, Kluwer Acad. Pub., Fevrier 1993, pp. 167-188.

[Lev05] Marc Levine, Mixed motives, Handbook of K-Theory (E.M Friedlander and D.R. Grayson, eds.), vol. 1, Springer-Verlag, 2005, pp. 429-535.

[Lev11] Marc Levine, Tate motives and the fundamental group., Srinivas, V. (ed.), Cycles, motives and Shimura varieties. Proceedings of the international colloquium, Mumbai, India, January 3-12, 2008. New Delhi: Narosa Publishing House/Published for the Tata Institute of Fundamental Research. 265-392 (2011)., 2011.

[LV12] Jean-Louis Loday and Bruno Vallette, Algebraic operads, vol. 346, Springer-Verlag, 2012 .

[Mil12] Joan Millès, The Koszul complex is the cotangent complex, Int. Math. Res. Not. IMRN (2012), no. 3, 607-650. MR 2885984

[Ree58] Rimhak Ree, Lie elements and an algebra associated with shuffles, Ann. of Math. (2) 68 (1958), 210-220.

[Reu93] Christophe Reutenauer, Free Lie algebras, London Mathematical Society Monographs. New Series, vol. 7, The Clarendon Press Oxford University Press, New York, 1993, Oxford Science Publications. MR 1231799 (94j:17002) 
[Sou12] Ismael Soudères, Cycle complex over $\mathbb{P}^{1}$ minus 3 points : toward multiple zeta values cycles., http://arxiv.org/abs/1210.4653, 2012.

[Sou13] Ismaël Soudères, Multiple zeta value cycles in low weight, http://arxiv.org/abs/1302.2462, 2013.

[Spi] Markus Spitzweck, Some constructions for voevodsky's triangulated categories of motives, preprint.

[SW11a] Dev Sinha and Benjamin Walter, Lie coalgebras and rational homotopy theory, I: graph coalgebras, Homology Homotopy Appl. 13 (2011), no. 2, 263-292. MR 2861231

[SW11b] Lie coalgebras and rational homotopy theory, I: graph coalgebras, Homology Homotopy Appl. 13 (2011), no. 2, 263-292.

[Voe00] V. Voevodsky, Triangulated category of motives over a field, Cycles, transfers, and motivic homology theories, Annals of Math. Studies, vol. 143, Princeton University Press., 2000.

[VSF00] Vladimir Voevodsky, Andrei Suslin, and Eric M. Friedlander, Cycles, transfers, and motivic homology theories, Annals of Mathematics Studies, vol. 143, Princeton University Press, Princeton, NJ, 2000. MR 1764197 (2001d:14026)

[Zag91] Don Zagier, Polylogarithms, Dedekind zeta functions and the algebraic K-theory of fields, Arithmetic algebraic geometry (Texel, 1989), Progr. Math., vol. 89, Birkhäuser Boston, Boston, MA, 1991, pp. 391-430.

Max-Planck-Insititut für Mathematik, Bonn, Vivatsgasse 7,, 53111 Bonn,, GerMANY,SOUDERES@MPIM-BONN.MPG.DE 